\title{
An Integrated Neural Decoder of Linguistic and Experiential Meaning
}

\author{
Andrew James Anderson, ${ }^{1,8}$ - Jeffrey R. Binder, ${ }^{2}$ Leonardo Fernandino, ${ }^{2}$ Colin J. Humphries, ${ }^{2}$ Lisa L. Conant, ${ }^{2}$ \\ (D)Rajeev D.S. Raizada, ${ }^{3}$ Feng Lin, ${ }^{1,3,4,5,8,9}$ and $\mathbb{D}^{\circ}$ Edmund C. Lalor ${ }^{1,6,7,8}$ \\ ${ }^{1}$ Department of Neuroscience, University of Rochester, Rochester, New York 14642, ${ }^{2}$ Department of Neurology, Medical College of Wisconsin, Milwaukee, \\ Wisconsin 53226, ${ }^{3}$ Department of Brain and Cognitive Sciences, University of Rochester, Rochester, New York 14627, ${ }^{4}$ School of Nursing, University of \\ Rochester, Rochester, New York 14642, ${ }^{5}$ Department of Psychiatry, University of Rochester, Rochester, New York 14642, ${ }^{6}$ Department of Biomedical \\ Engineering, University of Rochester, Rochester, New York 14627, ${ }^{7}$ School of Engineering, Trinity Centre for Bioengineering, and Trinity College Institute of \\ Neuroscience, Trinity College Dublin, Dublin 2, Ireland, ${ }^{8}$ Del Monte Institute for Neuroscience, University of Rochester, Rochester, NY 14642 , and \\ ${ }^{9}$ Department of Neurology, University of Rochester, Rochester, NY 14642
}

The brain is thought to combine linguistic knowledge of words and nonlinguistic knowledge of their referents to encode sentence meaning. However, functional neuroimaging studies aiming at decoding language meaning from neural activity have mostly relied on distributional models of word semantics, which are based on patterns of word co-occurrence in text corpora. Here, we present initial evidence that modeling nonlinguistic "experiential” knowledge contributes to decoding neural representations of sentence meaning. We model attributes of peoples' sensory, motor, social, emotional, and cognitive experiences with words using behavioral ratings. We demonstrate that fMRI activation elicited in sentence reading is more accurately decoded when this experiential attribute model is integrated with a text-based model than when either model is applied in isolation (participants were 5 males and 9 females). 0 ur decoding approach exploits a representation-similarity-based framework, which benefits from being parameter free, while performing at accuracy levels comparable with those from parameter fitting approaches, such as ridge regression. We find that the text-based model contributes particularly to the decoding of sentences containing linguistically oriented "abstract" words and reveal tentative evidence that the experiential model improves decoding of more concrete sentences. Finally, we introduce a cross-participant decoding method to estimate an upper bound on model-based decoding accuracy. We demonstrate that a substantial fraction of neural signal remains unexplained, and leverage this gap to pinpoint characteristics of weakly decoded sentences and hence identify model weaknesses to guide future model development.

Key words: concepts; fMRI; lexical semantics; multivoxel pattern analysis; semantic model; sentence comprehension

\section{Significance Statement}

Language gives humans the unique ability to communicate about historical events, theoretical concepts, and fiction. Although words are learned through language and defined by their relations to other words in dictionaries, our understanding of word meaning presumably draws heavily on our nonlinguistic sensory, motor, interoceptive, and emotional experiences with words and their referents. Behavioral experiments lend support to the intuition that word meaning integrates aspects of linguistic and nonlinguistic "experiential" knowledge. However, behavioral measures do not provide a window on how meaning is represented in the brain and tend to necessitate artificial experimental paradigms. We present a model-based approach that reveals early evidence that experiential and linguistically acquired knowledge can be detected in brain activity elicited in reading natural sentences.

\section{Introduction}

Humans' knowledge of historical events, theoretical concepts, and fiction is acquired through language. While a considerable body of linguistic information is stored in text and media repositories, the meaning of language is a biological construct, instan-

paper; J.R.B., L.F., C.J.H., and L.L.C. contributed unpublished reagents/analytic tools; J.R.B., L.F., R.D.S.R., F.L., and E.C.L. edited the paper.

This work was supported in part by University of Rochester Medical Center Schmitt Program on Integrative Neuroscience Award and the Intelligence Advanced Research Projects Activity via Air Force Research Laboratory 
tiated in our brains during comprehension. Recent advances in neuroimaging technology, big data, and computational modeling have led to an ability to decode brain activity associated with linguistic meaning. The dominant decoding approach achieves this using only text-based information. Word meaning is modeled as a vector of values reflecting how often each word cooccurred with other words across a huge body of text (Lund and Burgess, 1996; Landauer and Dumais, 1997; Mikolov et al., 2013; Pennington et al., 2014). Despite never having experienced walking or eating, the model "learns" that walk and eat mean different things because they appear in different textual contexts, but that walking relates to legs/shoes and that eating relates to hunger/ food because these words frequently co-occur (and end up with similar semantic vectors). This approach supports the construction of conceptual knowledge hierarchies (e.g., a dragonfly is an insect is an animal) (Fu et al., 2014), and enables some level of analogical reasoning (e.g., Einstein is to scientist as Picasso is to painter) (Mikolov et al., 2013). By registering model and brain activity for corresponding words, and mapping between the two, brain activity for new words, sentences and narratives can be predicted and decoded (Mitchell et al., 2008; Pereira et al., 2013; Wehbe et al., 2014; Huth et al., 2016a; de Heer et al., 2017; Pereira et al., 2018).

Behavioral experiments suggest that, in addition to linguistic experience, word meaning is shaped by nonlinguistic perceptual, motor, and interoceptive experiences (Paivio, 1971; Barsalou, 1999; Barsalou et al., 2008; Vigliocco et al., 2009, 2014; Andrews et al., 2009, 2014; Louwerse and Jeuniaux, 2010; Kousta et al., 2011; Riordan and Jones, 2011; Dove, 2014; Zwaan, 2014; Louwerse, 2018). Indirect evidence that sentence comprehension induces perceptual/motor simulations related to sentence content has been amassed in reaction time studies (Stanfield and Zwaan, 2001; Glenberg and Kaschak, 2002; Zwaan et al., 2002; Kaschak et al., 2005, 2006; Connell, 2007; Glenberg et al., 2008; Winter and Bergen, 2012; Zwaan and Pecher, 2012; Speed and Vigliocco, 2014). However, there is little direct neural evidence concerning how linguistic and nonlinguistic "experiential" sources of knowledge are encoded in brain activity (but see Anderson et al., 2015; Wang et al., 2018). The ability to estimate linguistic and nonlinguistic contributions to neural representations of meaning is necessary to fully characterize human language and related clinical conditions (Patterson et al., 2007; Fernandino et al., 2013; Ralph et al., 2017; Anderson and Lin, 2019; Bruffaerts et al., 2019). Critically, neural measures can also help provide a window on natural language comprehension, removing the necessity for artificial behavioral response tasks (which may perturb linguistic systems), and bespoke stimulus materials (that may not reflect natural language) (for related discussion, see Hamilton and Huth, 2018; Hasson et al., 2018).

We reveal initial evidence that nonlinguistic experiential knowledge can be detected in brain activity elicited in sentence reading by combining an experiential attribute model (Binder et al., 2016) with a state-of-the-art text-based semantic model (Pennington et al., 2014) to enhance decoding of a large fMRI dataset. The experiential model is based on peoples' ratings of their sensory/motor/affective/cognitive experiences with words and their

Grant FA8650 -14-C-7357 and National Science Foundation Career Award 1652127. We thank three reviewers for insightful comments and time; Xixi Wang for assistance in generating brain images; and Douwe Kiela and Katrin Erk for models used in supporting analyses.

The authors declare no competing financial interests.

Correspondence should be addressed to Andrew James Anderson at aander41@ur.rochester.edu.

https://doi.org/10.1523/JNEUROSCI.2575-18.2019

Copyright $\odot 2019$ the authors referents (building on Cree and McRae, 2003; Vinson et al., 2003; Lynott and Connell, 2013). While experiential models have provided a basis for neural decoding (Chang et al., 2011; Fernandino et al., 2015, 2016; Anderson et al., 2017a, 2019; Wang et al., 2017; Yang et al., 2017), it has never been clear whether and how textbased and experiential approaches complement one other. We newly demonstrate that text-based and experiential models differentially contribute to decoding sentences that do/do not contain linguistically oriented "abstract" words. Finally, we introduce a cross-participant decoding method to estimate the room for improvement in model-based decoding and pinpoint model weaknesses for future development.

\section{Materials and Methods}

\section{Overview}

We reanalyzed an fMRI dataset scanned as 14 people read 240 sentences describing everyday situations (Anderson et al., 2017a) (and summarized below). Sentences were 3 to 9 words long and formed from 242 different content words. Ten participants saw the set of sentences repeated 12 times in total (randomly shuffled each time), and the remaining 4 participants who attended half the number of visits saw the sentences 6 times. Following standard fMRI preprocessing steps, each sentence presentation was represented as a single fMRI volume (there were 12 replicate volumes per sentence for 10 participants and 6 replicates for the remaining 4 participants). Analyses were focused on a "semantic network" of 22 anatomical ROIs that had been detected in previous analyses of the same data (Anderson et al., 2019) testing for regional sensitivity to experiential semantic features associated with words with different grammatical roles. ROIs included left temporal, inferior parietal, inferior/ superior frontal cortex as well as some right hemispheric homologs (illustrated later in Fig. 6). These regions have well-established associations with semantic processing (e.g., Binder et al., 2009; Binder and Desai, 2011) and broadly adhere to the "language network" identified by Fedorenko and Thompson-Schill (2014). fMRI activation across the network of brain regions was then decoded using a text-based model, an experiential model, and the two models integrated as detailed below. We refer to the integrated text/experiential approach as "multimodal" to reflect the combination of linguistic information with behavioral ratings. Although to dispel any confusion, the experiential model serves as a proxy for knowledge acquired through multiple modalities of experience, just each modality is estimated through the same rating procedure.

\section{Materials}

All sentences were preselected as experimental materials for the Knowledge Representation in Neural Systems project (Glasgow et al., 2016) (www.iarpa.gov/index.php/research-programs/krns), sponsored by the Intelligence Advanced Research Projects Activity. The stimuli consisted of 240 written sentences containing 3-9 words and 2-5 (mean \pm SD $=$ $3.33 \pm 0.76)$ content words, formed from different combinations of 141 nouns, 62 verbs, and 39 adjectives ( 242 words). The sentences are listed in full in Anderson et al. (2017a) and Anderson et al. (2019). Sentences were in active voice and consisted of a noun phrase followed by a verb phrase in past tense, with no relative clauses. Most sentences (200 of 240) contained an action verb and involved interactions between humans, animals, and objects, or described situations involving different entities, events, locations, and affective connotations. The remaining 40 sentences contained only a linking verb ("was"). Each word occurs a mean \pm SD $(3.3 \pm 1.7$; range $1-7)$ times throughout the entire set of sentences and co-occurs with $8.1 \pm 4.3$ (1-19) other unique words. The same two words rarely co-occur in more than one sentence, and 213 of 242 words never co-occur more than once with any other single word. Forty-two sentences contained instances of words not found in any of the other 239 sentences, and 3 of these sentences contained 2 unique words. There were thus 45 words that occurred in only one sentence, of which 29 were nouns, 7 were verbs, and 9 were adjectives.

\section{Participants}

Participants were 14 healthy, native speakers of English ( 5 males, 9 females; mean age 32.5 years, range $21-55$ years) with no history of neuro- 
logical or psychiatric disorders. All were right-handed according to the Edinburgh Handedness Inventory (Oldfield, 1971). Participants received monetary compensation and gave informed consent in conformity with the protocol approved by the Medical College of Wisconsin Institutional Review Board.

\section{Procedure}

Participants took part in either 4 or 8 scanning visits. The mean interval between sessions was $3.5 \mathrm{~d}(\mathrm{SD}=3.14 \mathrm{~d})$. The range of the intervals between first and last visits was 15-43 d. In each visit, the entire list of sentences was presented 1.5 times, resulting in 12 presentations of each sentence over the 8 visits in 10 participants, and 6 presentations over 4 visits in 4 participants. Each visit consisted of 12 scanning runs, each run containing 30 trials (one sentence per trial) and lasting $\sim 6 \mathrm{~min}$. The presentation order of each set of 240 sentences was randomly shuffled.

The stimuli were back-projected on a screen in white Courier font on a black background. Participants viewed the screen while in the scanner through a mirror attached to the head coil. Sentences were presented word-by-word using a rapid serial visual presentation paradigm. Nouns, verbs, adjectives, and prepositions were presented for $400 \mathrm{~ms}$ each, followed by a $200 \mathrm{~ms}$ interstimulus interval. Articles ("the") were presented for $150 \mathrm{~ms}$ followed by a $50 \mathrm{~ms}$ interstimulus interval. Mean sentence duration was $2.8 \mathrm{~s}$. Words subtended an average horizontal visual angle of $\sim 2.5^{\circ}$. A jittered intertrial interval, ranging from 400 to $6000 \mathrm{~ms}$ $($ mean $=3200 \mathrm{~ms})$, was used to facilitate deconvolution of the BOLD signal. Participants were instructed to read the sentences and think about their overall meaning. They were told that some sentences would be followed by a probe word, and that in those trials they should respond whether the probe word was semantically related to the overall meaning of the sentence by pressing one of two response keys ( $10 \%$ of trials contained a probe). Participants' mean accuracy was $86 \%$ correct, with a minimum accuracy of $81 \%$. Participants were given practice with the task outside the scanner with a different set of sentences. Response hand was counterbalanced across scanning visits.

\section{MRI parameters and preprocessing}

MRI data were acquired with a whole-body 3T GE 750 scanner at the Center for Imaging Research of the Medical College of Wisconsin using a GE 32-channel head coil. Functional T2*-weighted EPIs were collected with $\mathrm{TR}=2000 \mathrm{~ms}, \mathrm{TE}=24 \mathrm{~ms}$, flip angle $=77^{\circ}, 41$ axial slices, $\mathrm{FOV}=$ $192 \mathrm{~mm}$, in-plane matrix $=64 \times 64$, slice thickness $=3 \mathrm{~mm}$, resulting in $3 \times 3 \times 3 \mathrm{~mm}$ voxels. T1-weighted anatomical images were obtained using a $3 \mathrm{D}$ spoiled gradient-echo sequence with voxel dimensions of $1 \times$ $1 \times 1 \mathrm{~mm}$. fMRI data were preprocessed using AFNI (Cox, 1996). EPI volumes were corrected for slice acquisition time and head motion. Functional volumes were aligned to the T1-weighted anatomical volume, transformed into a standardized space (Talairach and Tournoux, 1988), and smoothed with a $6 \mathrm{~mm}$ FWHM Gaussian kernel. The data were analyzed using a GLM with a duration-modulated HRF, and the model included one regressor for each sentence. Neural activity was modeled as a gamma function convolved with a square wave with the same duration as the presentation of the sentence, as implemented in AFNI's 3dDeconvolve with the option dmBLOCK. Duration was coded separately for each individual sentence. Finally, a single sentence-level fMRI representation was created for each unique sentence by taking the voxelwise mean of all replicates of the sentence.

\section{Experimental design and statistical analysis}

Semantic models. Analyses used the following two semantic models of word meaning. As a proxy for the representational structure that can be acquired the distributional statistics of words in language we used "GloVe" (Pennington et al., 2014). GloVe is a freely downloadable textbased semantic model that represents individual words as 300 dimensional floating point vectors derived by factorizing a word co-occurrence matrix (vocabulary size is 2.2 million words, and co-occurrences were measured across 840 billion tokens from Common Crawl, https:// commoncrawl.org). GloVe in particular was used because it yielded state-of-the-art performance decoding fMRI activation associated with sentences in Pereira et al. (2018) "universal neural decoder of linguistic meaning," although we found there to be relatively minor differences between using GloVe and other models, such as "word2vec" (Mikolov et al., 2013) (see also Supporting analysis: persistence of multimodal advantage using different text-based models).

As a proxy for the representational structure that can be acquired from direct experience with the world, we used an experiential attribute model (Binder et al., 2016). This model represents words in terms of human ratings of their degree of association with different attributes of experience (e.g., "On a scale of 0 to 6 , to what degree do you think of a banana as having a characteristic or defining color?"). Ratings were collected via Amazon Mechanical Turk for a total of 65 attributes spanning sensory, motor, affective, spatial, temporal, causal, social, and abstract cognitive experiences. Ratings for each attribute were averaged across workers to derive a single 65 dimensional vector for each word. As such, this model broadly aligns with "embodiment" theories that posit representations of word meaning reflect a summarization of the brain states involved in experiencing that word, partially reenacted across sensory/motor/affective/cognitive subsystems (e.g., Barsalou et al., 2008; Glenberg, 2010; Pulvermüller, 2013; Binder et al., 2016). This same experiential model has previously been used as the basis for predicting and decoding the same fMRI dataset as the current study (Anderson et al., 2017a, 2019).

Some overlap in the semantic information content of text-based and experiential models is very much expected (see also Riordan and Jones, 2011). Because text describes worldly experiences, we expect it to partially capture the structure of experiential knowledge. On the flipside, the experiential attribute model seeks to comprehensively model experiential knowledge; and of course, language contributes to our experience. Beyond this, the experiential model was itself built through a linguistically guided rating procedure. However, systematic differences between the models are also expected. This is in part because a lot of experiential information goes unstated in natural verbal communication. For instance, borrowing an example from Bruni et al. (2014), it is rarely useful to communicate the color of bananas because it is obvious to all those with experience of bananas. Likewise, it would be unusual to specify that dropping things involves movement. Consequently, while an analysis of natural text may indicate that a banana is an edible berry, it may not capture the dominance of color as a perceptual attribute. Therefore, despite being derived via language, attribute ratings can potentially anchor to experiential neural systems and access information that would not otherwise have been reported or experienced in natural verbal communication. Conversely, the experiential attribute model as it stands may be less well suited to capturing the meaning of so-called abstract words, which tend to be more amenable to verbal description in terms of their relationships with other words (e.g., "fiction" is an imaginary story), rather than through physical example (e.g., being presented with a cat).

We consider the extent to which experiential information is available in text (and language) to be an empirical question. For instance, representational similarity analysis (RSA) (Kriegeskorte et al., 2008) can be applied to compare the information structure of the text based to the experiential models. This yields a statistically significant correlation coefficient of $\rho=0.2, p<10^{-6}$ (reflecting Spearman correlation between the below diagonal triangles of interword Pearson correlation matrices derived using the text-based and experiential models, $n=29,161$ correlation coefficients per triangle as computed from 242 words). A related regression analysis conducted by Utsumi (2018) demonstrated that textbased models were weakly capable of predicting spatial, temporal, and affective attributes of the current experiential model. However, demonstrating that text-based models do not contain aspects of experiential information does not also entail that the missing information is relevant for explaining semantic brain activity. Consequently, we conduct our forthcoming analyses by testing for brain activity that is explainable using the experiential model but not using the text-based model and vice versa (see also Anderson et al., 2015; Popov et al., 2018). This approach takes the assumption that the text-based model accurately captures all semantic information that can be extracted from text alone. While we acknowledge that this is a strong assumption that is not likely to have been strictly met here, we believe that current models are sufficiently advanced to begin to segregate experiential from linguistic aspects of semantic representation in brain activity (see also the Discussion). 


\section{Neural, experiential and text-based sentences}
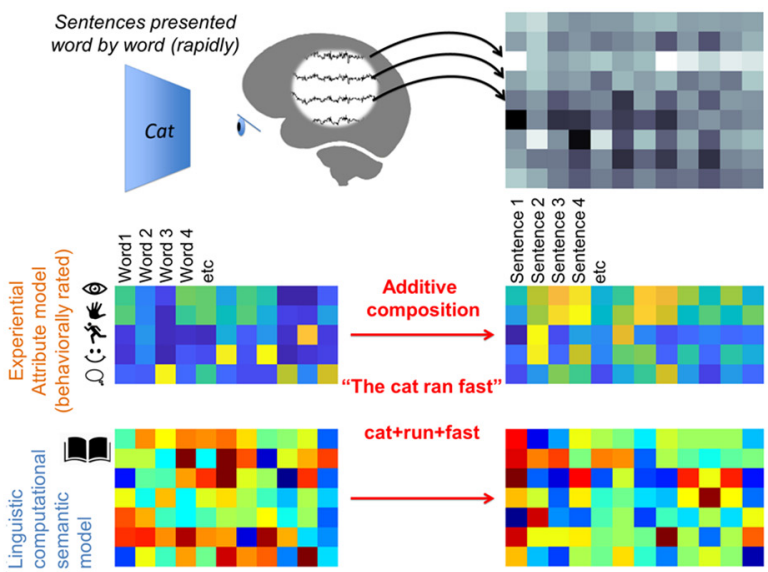

\section{Re-representation in common similarity space}

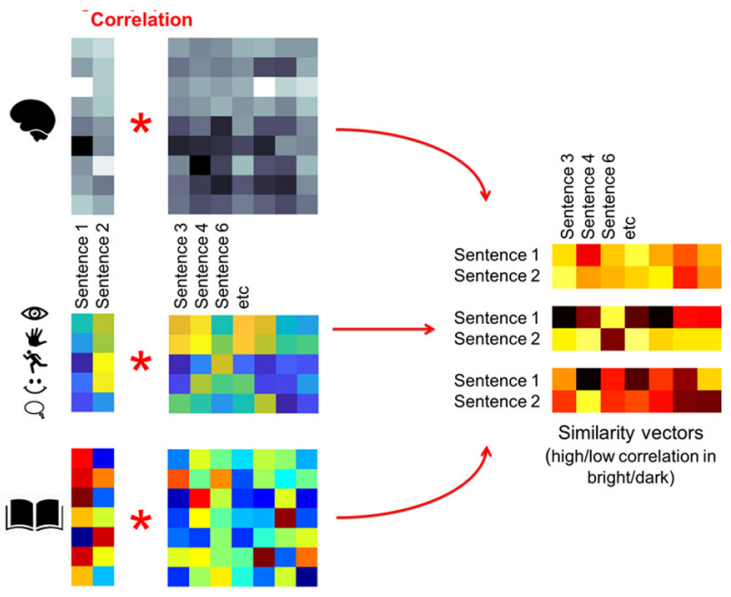

2. Cross validation training/test split

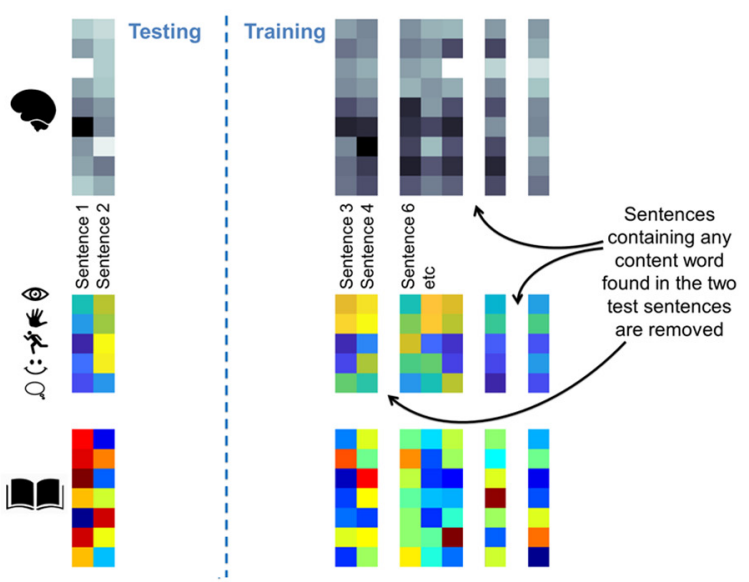

4. Multimodal model-based decoding

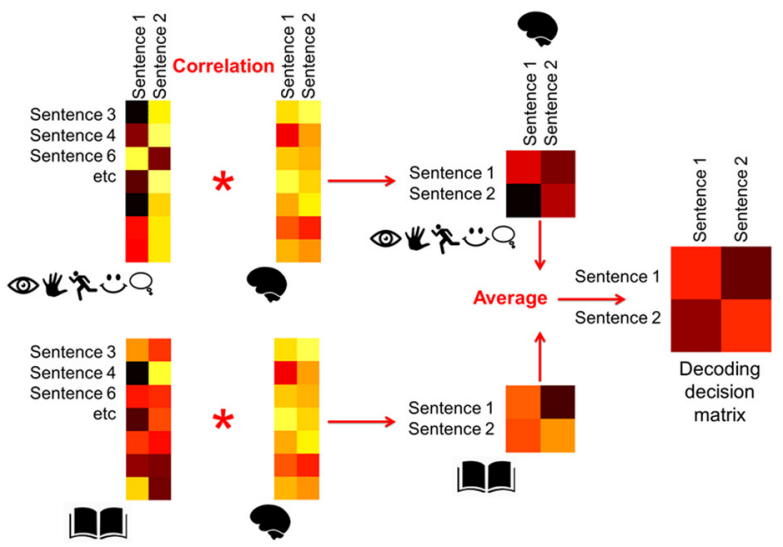

Figure 1. Representational similarity-based decoding algorithm set up to support multiple model-based decoding. Multimodal model combination takes place in Stage 4 by averaging $2 \times 2$ decoding decision matrices generated by the different models. An alternative approach would have been to pointwise average together the two similarity vectors for the experiential model with those of the text-based model in Stage 3. This was disfavored to avoid having to introduce an extra normalization step to deal with correlation coefficients arising from the different models being on different scales (correlation coefficient magnitudes tend to diminish as the number of features correlated becomes large, and here the experiential and text-based models widely differ in the number of features: 65 and 300, respectively). This problem is naturally dealt with in Stage 4 because the $2 \times 2$ decision matrices are based on correlations between similarity vectors that are all matched in their dimensions. Each red asterisk corresponds to Pearson's correlation coefficient.

Modeling sentences by summing word-level semantic vectors. To turn word-level semantic vectors into representations of sentences, we identified all constituent content words in each sentence, and then pointwise summed together these semantic vectors (Fig. 1). Although such additive composition is obviously an oversimplification that neglects the effects of word order, syntax, and morphology, it has endured as a practically successful technique in both computational linguistics (Mitchell and Lapata, 2010; Kiela and Clark, 2014), and fMRI analyses (Anderson et al., 2017a, 2019; Wang et al., 2017; Yang et al., 2017; Pereira et al., 2018). Indeed, attempts to incorporate other linguistic factors, such as syntax, into models have yet to make appreciable difference to neural decoding performance (Pereira et al., 2018; Anderson et al., 2019).

Representational similarity-based neural decoding setup. To decode fMRI activation, we applied the representational similarity (see also Kriegeskorte et al., 2008), decoding framework introduced by Anderson et al. (2016, 2017b) and further extended here to support simultaneous multimodel/multi-ROI/multiparticipant decoding of sentences. This was a considered departure from the more commonplace strategy of using multiple regression to map between model and brain (Mitchell et al., 2008; Chang et al., 2011; Sudre et al., 2012; Pereira et al., 2013, 2018; Wehbe et al., 2014; Fernandino et al., 2015, 2016; Huth et al., 2016a; Anderson et al., 2017a, 2019; Wang et al., 2017; Yang et al., 2017). The main reason for choosing the similarity-based approach over (ridge) regression here was for simplicity: to avoid repeating the analyses multiple times over with different regularization penalties and the need to introduce a decision over which penalty to use. For the current analyses, this would complicate the process of integrating information across models, ROIs, and individuals (because, in each case, there would be multiple results associated with the different penalties and multiple decisions to be made). The current focus on the similarity-based approach should not be misconstrued as a claim that similarity-based methods are superior to regression, and we report on some strengths and weaknesses of the two approaches in a supporting analysis using ridge regression (see Results; Fig. 10). Other comparative analyses are in Anderson et al. (2016) and Bulat et al. (2017).

fMRI data were decoded according to a commonly used leave-2-itemout cross-validation procedure (Mitchell et al., 2008; Chang et al., 2011; Sudre et al., 2012; Pereira et al., 2013, 2018; Wehbe et al., 2014; Anderson et al., 2016, 2017a, 2019; Wang et al., 2017; Yang et al., 2017). At each cross-validation iteration, the 240 sentences were split into a test set of 2 sentences and a training set of 238 sentences. Then both fMRI and model data for any of the 238 training sentences that contained any content word within the 2 test sentences were deleted from the training data (Fig. 1 , Stage 2). This was to enable testing of how well the approach generalized to decoding novel fMRI sentences using sentence vectors built from an entirely novel set of semantic vectors. The mean \pm SD number of 
sentences in the training set for each iteration was $218 \pm 5$, containing a mean \pm SD of $232 \pm 2$ words. Model and fMRI data corresponding to the training set was featurewise/voxelwise $z$-scored. Model and fMRI test sentence data were likewise normalized by subtracting the featurewise/ voxelwise mean and dividing by the featurewise/voxelwise $\mathrm{SD}$ of the training data.

Because not all fMRI voxels contain informative signal, we estimated which ones were likely to be informative using a commonly used strategy (e.g., Mitchell et al., 2008; Chang et al., 2011; Pereira et al., 2013; Anderson et al., 2015, 2016, 2017b; Wang et al., 2017; Yang et al., 2017). For each participant, and separately for each ROI, we took each of the 12 (or 6) fMRI runs through the entire set of sentences, selected only the 218 (or so) training sentences from this, and then voxelwise correlated each unique pair of runs together. For the 10 participants with 12 runs, this left 66 pairwise correlation coefficients per voxel; and for the 4 participants with 6 runs, this left 15 pairwise correlation coefficients per voxel. A single score was assigned to each voxel by taking the mean of these ( 66 or 15) correlation coefficients. The 50 voxels with the largest mean value per ROI were selected for analysis. This choice of 50 voxels was ultimately arbitrary though guided by previous work (e.g., Anderson et al., 2013, 2015, 2017b). In subsequent post hoc analyses (reported later), the interpretation of results was found to be unchanged when using 100 voxels; however, in both cases, voxel selection systematically improved decoding accuracies over no voxel selection at all.

FMRI decoding was accomplished by independently rerepresenting both model and fMRI test data in a common representational similarity space, and then matching model to fMRI sentences in this space. For each fMRI/model dataset in turn, we correlated the two test sentence vectors with each one of the 218 (or so) training sentence vectors. This enabled us to newly represent every single test sentence (whether model or fMRI) as a similarity vector of 218 (or so) correlation coefficients (Fig. 1, Stage 3). We transformed all correlation coefficients in all similarity vectors using Fisher's $r$-to- $z$ (arctanh), as is a customary treatment comparing correlation values (although this had only a marginal effect on the current results). Then decoding was achieved by cross-correlating the two model similarity vectors with the two fMRI similarity vectors (Fig. 1, Stage 4). This resulting $2 \times 2$ matrix of correlation coefficients was $r$-to- $z$ transformed (arctanh), and this constituted the "decoding decision." This was evaluated as correct (and scored as a 1 as opposed to zero) only if the sum of $z$-transformed correlations between the correctly matched model versus fMRI test sentence pair (Fig. 1, matrix diagonal, Stage 4), exceeded the sum for the incongruent pair (Fig. 1, antidiagonal, Stage 4). Decoding was repeated for all possible unique pairs of the 240 sentences $(28,680$ cross-validation iterations in total), and the mean score used as the final metric of decoding accuracy. When operating at random (e.g., if the fMRI data contained no semantic signal, or the semantic vectors did not reflect meaning), a mean decoding accuracy of 0.5 is expected. Permutation testing was applied to test whether decoding accuracies were significantly better than chance (by randomly shuffling semantic model sentences and repeating the entire cross-validation analysis 1000 times over, as described by Anderson et al., 2017b). Typically, final decoding accuracies $>0.56$ were significant at the $p=0.05$ level ( $95 \%$ of metrics derived in analyzing randomly shuffled sentences were less than or equal to 0.56 ).

For each participant, $2 \times 2$ decoding decisions were computed in parallel for all 22 ROIs and each of the 2 models. To generate a single decoding decision corresponding to the entire semantic network of all 22 ROIs, we applied an "ensemble averaging" strategy and pointwise averaged together decision matrices across the 22 ROIs (Fig. 2, top row). We use this network-level decoding estimate as the basis for our main analyses but also report results for individual ROIs.

In a similar vein, we used ensemble averaging as the basis for testing whether models have complementary strengths in decoding. If the models have complementary strengths, then integrating their decisions together as an ensemble will counteract an individual model's weaknesses and boost overall decoding accuracy. Multimodal model integration was achieved by pointwise averaging decision matrices associated with the different models as illustrated in Figure 1 (Stage 4). In all ensemble averaging tests (whether integrating ROIs and/or models), decoding decision
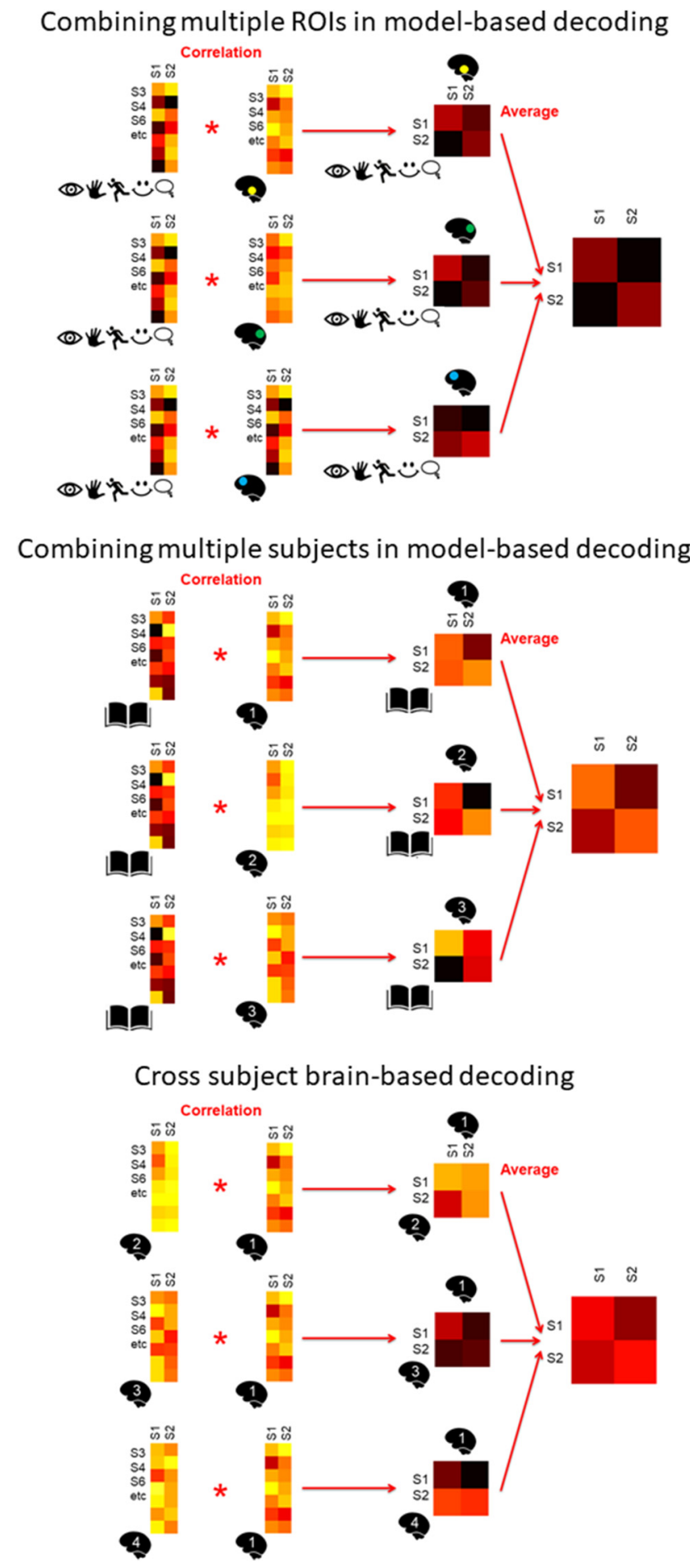

Figure 2. Representational similarity-based algorithm setups for ensemble decoding. Top, Model-based decoding of multiple brain regions in the same participant (see also results in Figs. $4-6,8,9$, and 11). Middle, Model-based decoding of multiple participants (see also results in Fig. 5). Bottom, Cross-subject decoding (see also results in Fig. 8). Each red asterisk corresponds to Pearson's correlation coefficient.

matrices were scored as correct precisely as before by testing whether correlations on the diagonal were greater than the antidiagonal. To produce a final metric, scores were then averaged across all 28,680 crossvalidation iterations. Importantly, this ensemble averaging strategy is not guaranteed to produce equivalent or better final accuracies than the strongest model of the pair (which would limit its applicability for testing for a multimodal decoding advantage). Specifically, if one model is suf- 
ficiently noisy, then the final multimodal decoding accuracy will be lower than the strongest model.

The entire cross-validation procedure described above was repeated for each ROI (22) within each participant (14) using both semantic models (2). Ensemble averaging of model decision matrices was used to derive multimodal decoding accuracies for each participant and ROI. Ensemble averaging of all 22 ROIs' decision matrices was used to generate a single network-level decoding accuracy for each participant and model combination (text, experiential, and multimodal). Differences in accuracy between different models were evaluated using $t$ tests, and $p$ values associated with multiple ROIs corrected for multiple comparisons according to false discovery rate (FDR) (Benjamini and Yekutieli, 2001).

Post hoc analyses partitioning the variance in neural similarity structure explained by each model. ROIs for which decoding accuracy was boosted through model integration were taken to post hoc analyses. We further estimated the unique contribution made by each model to explaining variance in the neural sentence similarity structure and what could be explained equally by both models. This analysis is inspired by de Heer et al. (2017) who partitioned the variance in heard speech fMRI data, which were unique to acoustic, articulatory, and semantic voxelwise encoding models and shared across them. In addition to using different models to de Heer et al. (2017), the forthcoming analysis differs in that it is conducted in representational similarity space. The analysis is illustrated in Figure 3.

The analysis was conducted on the entire similarity space defined by all 240 sentences as is a standard approach in RSA (Kriegeskorte et al., 2008). Sentence similarity matrices were computed for each participant, for each ROI, by intercorrelating the neural representations of the 240 sentences. In each case, this yielded a $240 \times 240$ correlation matrix. Pearson correlation was used, and the correlation coefficients were subsequently $r$-to- $z$-trans-

formed (arctanh). Before this, voxel selection was conducted to estimate the 50 informative voxels per ROI. Voxel selection used the same correlation-based approach as detailed above for the previous decoding analysis. However, in the case at hand, voxel selection was computed only once on all 240 sentences together (because the current RSA did not use cross-validation). To generate a single correlation matrix capturing the 22 ROI ensemble, the $22 r$-to- $z$-transformed correlation matrices corresponding to each ROI were pointwise averaged.

Correlation matrices for the text-based and experiential models were computed in the same fashion by intercorrelating the 240 sentences. Again, Pearson correlation was used, and all coefficients were $r$-to- $z$-transformed. Next, the unique information contained within every single correlation matrix was extracted by segmenting the below diagonal matrix triangle. Each triangle was then vectorized to create a 28,680 element similarity vector. Similarity vectors were subsequently normalized by $z$-scoring to support the forthcoming regression analysis.

We applied the set theoretic approach of de Heer et al. (2017) to estimate the variance in neural similarity structure that was explained by the union of both models, the shared variance that is equally explained by either model, and the variance solely accountable to one model (Fig. 3). The union $\left[R^{2}\right.$ Text $\cup$ Experiential $]$ was first estimated using a multiple regression in which the similarity vectors for both models were used as predictors and the neural similarity vector was the response variable. The variance associated with but not necessarily exclusive to the individual models ( $\left[R^{2}\right.$ Text $]$ and $\left[R^{2}\right.$ Experiential $]$ ) was estimated in two separate regression analyses. In each, a single similarity vector (associated with one model) was the predictor. To estimate the shared variance $\left[R^{2}\right.$ Text $\cap$ Experiential], we subtracted away the variance explained by the model union $\left[R^{2}\right.$ Text $\cup$ Experiential $]$ from the sum of the variance explained by the Text and Experiential models $\left(\left[R^{2}\right.\right.$ Text $]+\left[R^{2}\right.$ Experiential] $)$. Then, to estimate the variance solely accountable to the text and experiential models ([ $R^{2}$ Solely Text $]$ and $\left[R^{2}\right.$ Solely Experiential $]$ ), we subtracted the shared variance away from the variance explained by each model (e.g., $\left[R^{2}\right.$ Text $]-R^{2}$ Text $\cap$ Experiential $]$ ). To produce positive correlation coefficients from these measures, we took the square root of $R^{2}$ values as undertaken by de Heer et al. (2017). However, because variance/positive correlation measures do not facilitate testing whether individual models made significantly greater than zero contribution to explaining neural data (because they are always greater than or equal to zero), we also undertook a partial correlation analysis (see also Anderson et al., 2015; Wang et al., 2018). Here we computed the correlation between neural similarity structure and one model while controlling for the other model. To test the generality of partial correlation 

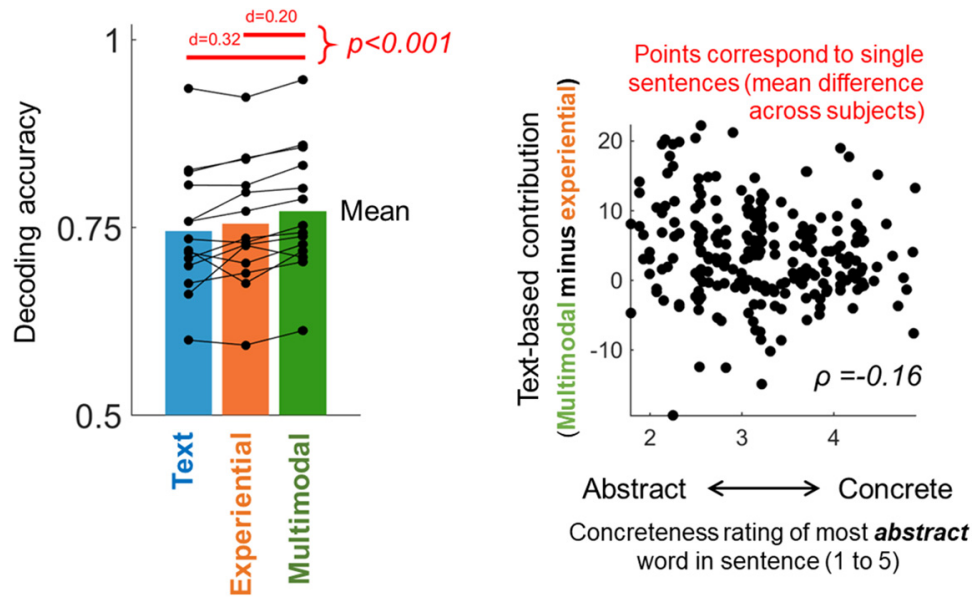

Figure 4. Integrating text-based and experiential models produces stronger decoding. Individual-level accuracies arising from decoding the 22 ROI ensemble (see Fig. 2, top row). The contribution of the text-based model to multimodal decoding was particularly pronounced for sentences containing abstract words (right). Effect sizes ( $d$ ) were estimated according to Dunlap et al. (1996) as $d=t \times(2 \times(1-r) / n)$, where $t$ is the $t$ statistic arising from the corresponding paired $t$ test, $r$ is Pearson correlation, and $n$ is the number of participants (14). $\rho$ corresponds to Spearman's correlation coefficient.

coefficients across participants, we compared them to zero using onesample $t$ tests $(n=14)$.

Code accessibility

MATLAB similarity-based decoding code is available on request from the corresponding author.

\section{Results \\ Integrating text-based and experiential semantic models produces stronger decoding than either alone}

To test for evidence that both linguistic and nonlinguistic experiential aspects of meaning were present in neural activation, we tested whether combining the two models together improved decoding accuracies above using either model in isolation. Decoding accuracies for both models and their combination are illustrated in Figure 4. $t$ tests revealed that, while there were no differences in decoding accuracy between the text-based and experiential models, when the models were combined, accuracies were significantly greater than for either model in isolation (difference between multimodal and text, $t=6.9, p<0.0001$; difference between multimodal and experiential, $t=4.8, p=0.0004$ ). This key result provides direct neural evidence that linguistic and experiential semantic information were present in brain activation elicited in sentence reading. Otherwise, decoding accuracies were significant for all participants and all models.

\section{Text-based model enhances discrimination of sentences containing abstract words}

We next examined the nature of the contribution made by the text-based and experiential model in more detail. An obvious area to anticipate divergence between models is for more linguistically oriented "abstract" words. These words do not directly correspond to "concrete" entities in the world that can be directly sensed. As such, an abstract word's meaning is languagedependent and most amenable to description in terms of other words, which could contribute different parts of that meaning (Brysbaert et al., 2014). Examples of words that are relatively abstract within the current dataset include: "negotiate," "agreement," "wealthy," "famous," and "clever." We hypothesized that the text-based model (which is built from word co-occurrence statistics) would make a particular contribution to multimodal decoding of sentences containing abstract words.

To test for an abstractness advantage associated with integrating the text-based model in decoding, we looked up concreteness ratings (Brysbaert et al., 2014) for each of the 242 content words in the sentence set. Each of the 240 sentences was then scored according to the following: (1) the concreteness of the least concrete (most abstract) word in the sentence; (2) the concreteness of the most concrete word in the sentence; and (3) the mean concreteness of all words in the sentence. Because the experimental sentences also varied across many other factors which through coincidence might be confounded with concreteness measures, we attempted to take these into account. Specifically, we additionally scored each sentence according to the least frequent, most frequent, and mean frequency of content words in the sentence (derived from log2 transformed SUBTLEX-US counts of Brysbaert and New, 2009), the number of words in each sentence, and the minimum, maximum, and mean word length (number of characters). After this, each sentence was represented with 10 measures (including the 3 concreteness measures).

We then identified how well each individual sentence was decoded using the different models. For each participant $(n=$ 14), this yielded a vector of 240 sentence decoding scores for the text-based model, the experiential model, and the multimodal combination. The maximum score attainable (and maximum value in the vector) was 239 , which could have been achieved if the corresponding sentence was successfully discriminated from all 239 other sentences during the entire leave-2-out crossvalidation analysis.

To estimate the independent contribution made by the textbased model to multimodal decoding, we pointwise subtracted the experiential model-based decoding scores for individual sentences away from corresponding multimodal model scores (and repeated for each participant). Positive scores arising from this subtraction indicate sentences that were better discriminated by integrating the text-based model, which in turn we hypothesized relate to a measure of sentence abstractness. To test this, we correlated (Spearman) each participant's vector of "boost" values with the three different sentence concreteness measures (leaving $14 \times 3$ correlation coefficients). We repeated these correlations for the other seven sentence measures (re: word frequencies and lengths of constituent words and the number of words in the sentence).

To test for the generality of positive correlations across participants, participants' correlation coefficients were $r$-to- $z$ transformed (arctanh) and then compared with zero using a onesample $t$ test $(n=14)$. $t$ tests were repeated on correlation coefficients associated with each of the 10 sentence measures. The resultant $10 p$ values were FDR-corrected. Only 1 of the $10 t$ tests yielded a statistically significant result. This was the test based on correlations between the concreteness rating of the most abstract word in the sentence and the decoding boost $(t=-3.7, p=0.04$, FDR-corrected). The mean correlation coefficient across participants was -0.08 . This provided evidence that the decoding ad- 


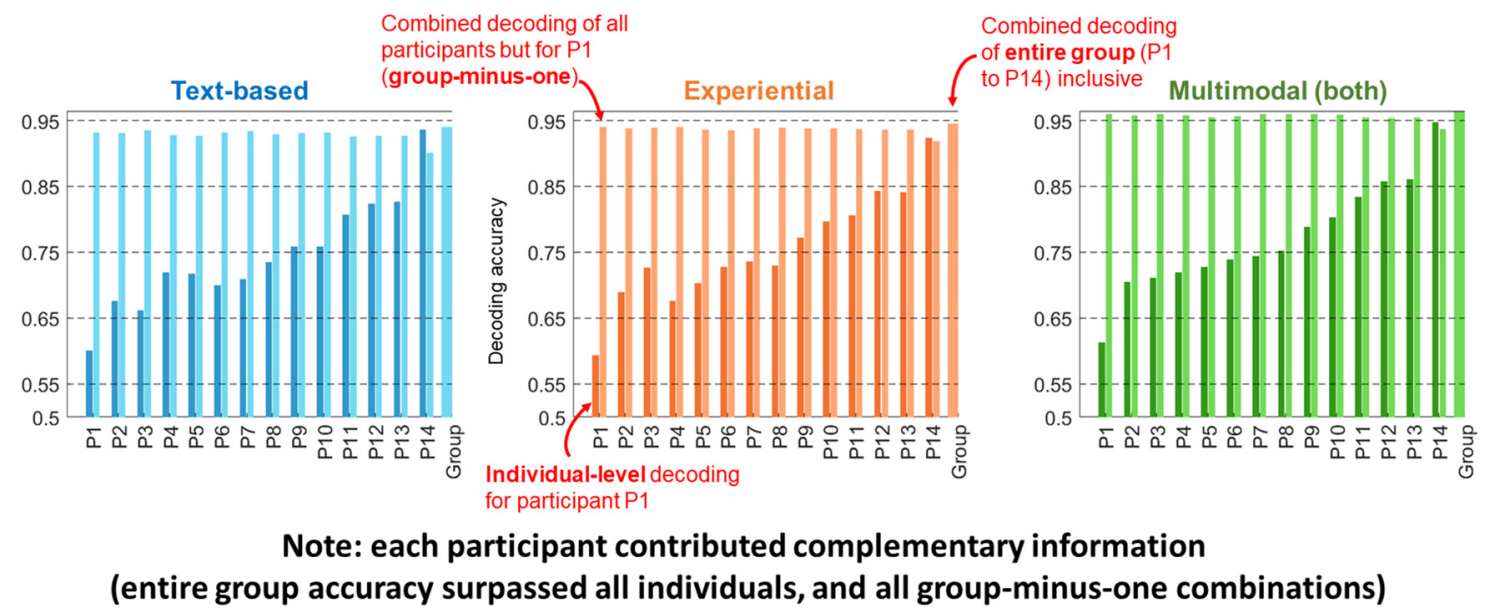

Figure 5. Decoding neural data at group level exploits cross-participant regularities. Model-based decoding accuracies at group level (Fig. 2, middle) and for each individual corresponding to all 22 ROIs decoded as an ensemble. Individual participants' decoding accuracies (dark) are plotted beside group-minus-one (light) decoding accuracies derived using all other participants. "Group" is all 14 participants combined.

vantage brought by integrating the text-based model was related to better discrimination of sentences containing abstract words. This relationship is illustrated in Figure 4 (the figure illustrates the mean accuracy boost per sentence across all 14 participants with associated correlation coefficient of -0.16 , whereas the above $t$ test was based on 14 individual-level correlation coefficients, with a mean value of -0.08).

In an attempt to gain an intuition of whether there was a common theme to the sentences containing abstract words that had received a decoding boost, we listed them. However, we were unable to confidently pinpoint any systematic pattern. The 10 sentences that received the greatest decoding boost are as follows with the most abstract word in each sentence in italics. The abstract word's concreteness rating $(\mathrm{C})$ and the mean decoding boost (B) associated with the sentence are in parentheses after the sentence. "The family was happy." $(\mathrm{C}=2.6, \mathrm{~B}=22)$; "The team celebrated." ( $\mathrm{C}=2.9, \mathrm{~B}=21)$; "The patient survived." $(\mathrm{C}=2.5$, $B=21)$; "The family survived the powerful hurricane." $(C=2.6$, $\mathrm{B}=20)$; "The pilot was friendly." $(\mathrm{C}=2.3, \mathrm{~B}=20)$; "The flood was dangerous." ( $\mathrm{C}=2.1, \mathrm{~B}=20)$; "The man lost the ticket to soccer." $(\mathrm{C}=2.3, \mathrm{~B}=20)$; "The jury watched the witness." $(\mathrm{C}=$ $4.1, \mathrm{~B}=19)$; "The council read the agreement." $(\mathrm{C}=2.2, \mathrm{~B}=18)$; "The artist shouted in the hotel." ( $\mathrm{C}=4.2 \mathrm{~B}=18)$. On face value, a commonality would appear to be that many of the sentences have affective connotations. However, such affective connotations were not exclusive to the boosted sentences. Indeed, three of the four sentences that were most disadvantaged by integrating the text-based model were also valenced. These four sentences were as follows: "The team lost the football in the forest." ( $\mathrm{C}=2.3 \mathrm{~B}=-19)$, "The teacher broke the small camera." ( $\mathrm{C}=3.2, \mathrm{~B}=-15)$, "The aggressive team took the baseball." ( $\mathrm{C}=2.5, \mathrm{~B}=-12.4)$, "The actor gave the football to the team." ( $\mathrm{C}=2.8, \mathrm{~B}=-12.5)$. We therefore presume simply that the text-based model helped explain neural signal reflecting the additional linguistic information exposed in accessing abstract words and integrating their meaning into sentences. However, in the future, it will also be valuable to consider stimuli that are more controlled in their content and less dominated by concrete sentences than the current dataset.

We next ran an analogous analysis attempting to understand the contribution of the experiential model, which we anticipated might be associated with concreteness. Sentence-wise decoding score vectors (length 240, maximum value 239) for the text-based model were subtracted from the multimodal model to generate an "accuracy boost" vector for each participant. Correlations between boost vectors and all 10 sentence measures were computed. While a significant positive correlation was detected with the concreteness of the most abstract word in the sentence: that is, the contribution of the experiential model was especially associated with concrete sentences without any abstract words (average correlation across participants $=-0.045, p=0.02$, disappointingly this result did not survive FDR correction for multiple comparisons and should be treated tentatively for the time being.

\section{Model-based neural decoding at group-level leverages cross-participant regularities}

A fundamental difference between either of the semantic models and individuals' fMRI data were that models were generic representations of concepts built from group-level information (either from texts written by many authors, or ratings given by many people), whereas fMRI activation captured snapshots of an individual's interpretation of a sentence at the particular time of scanning, and in the broader context of their own personal experiences. We therefore reasoned that combining individuals' fMRI data together as a group should expose regularities in semantic representations across individuals and lead to a stronger pattern match to the group-level models (Fig. 2, middle). Aside from this, the group-level combination should also iron out noise (whether this arises due to technological reasons or participants attention levels and/or compliance with the task). Either way, this should lead toward a less noisy comparison between model and fMRI data, albeit at the expense that the results may not generalize to individuals (though this has already been tested in Fig. 4).

Combining fMRI activation across participants is in general complicated by both anatomical and functional differences between individuals. While sophisticated "hyper-alignment' methods for combining group fMRI data exist (Haxby et al., 2011; Guntupalli et al., 2016), in the current case, it is also possible to combine individuals together as a group, by averaging together individual's decoding decision matrices (see Fig. 2, middle) in precisely the same way as we have combined models and ROIs.

Group-level decoding accuracies, illustrated in Figure 5, were unanimously greater than average individual-level decoding accuracies. For example, where the mean individual-level decoding 


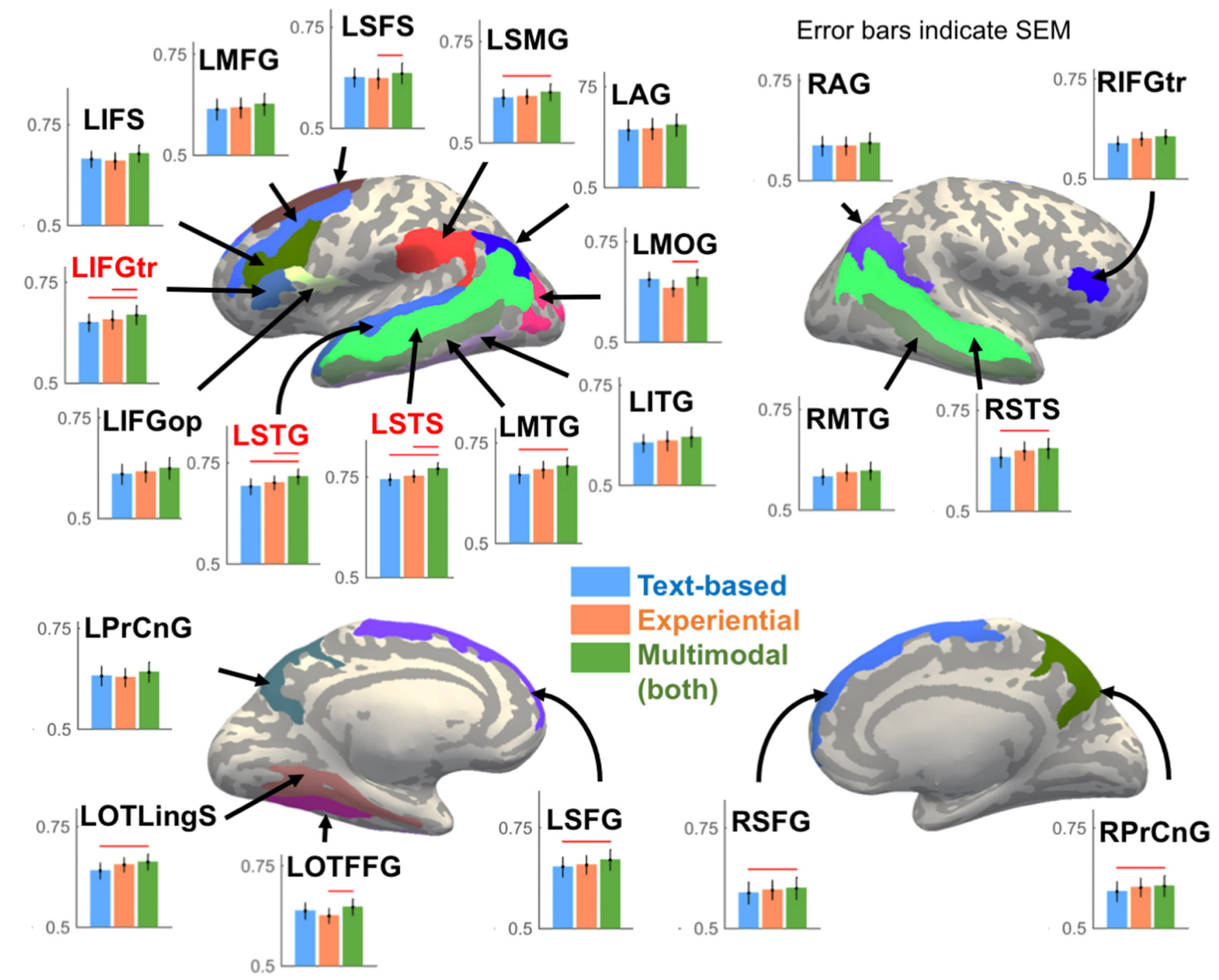

Error bars indicate SEM

Significant $(p<0.05)$ difference between connected model pair (paired t-test, FDR corrected)

Figure 6. Multimodal model integration improves decoding of superior temporal and inferior frontal regions. Data are mean \pm SEM decoding accuracies across 14 participants derived using the text-based and experiential model independently, and then when combined together (i.e., multimodal decoding; see Fig. 1, Stage 4).

accuracy was 0.77 , the group-level result was 0.97 (as a side note, although this may seem surprisingly high accuracy, this score is consistent with previous word-level decoding studies [Anderson et al., 2016, 2017b], and also reflects the high sensitivity of the leave-2-out test). To confirm the statistical significance of this effect, we used one-sample $t$ tests to test the individual-level decoding results against the single group-level score. The outcome was significant $(p<0.05)$ in every test following FDR correction (for all ROIs and for both models and their multimodal combination).

In conducting group-level analyses, it is possible that individual participants play a dominant role in results (an individual may have just happened to elicit semantic representations that match the models well, or have been particularly attentive to the task). To examine the influence of individual participants, we reran the group-level analysis holding out each individual from the group in turn. Group minus participant decoding accuracies are plotted next to the held-out participant in Figure 5. It became clear that one participant (P14) indeed played a dominant role relative to the other participants (the decoding accuracy for P14 was slightly greater than group-level decoding based on P1 to P13). However, when P14 was excluded from the group, decoding accuracies remained high (0.94), and this accuracy was substantially greater than individual results for P1 to P13. Therefore, although P14 played a dominant role, the group-level advantage persisted when this participant was excluded. Interestingly, the decoding accuracy for the entire group (all 14 participants) always exceeded every single individual-level decoding accuracy, and every group-minus-one decoding accuracy, which indicates that every single participant, including the poorest decoded (P1) beneficially contributed to the group-level decision.

\section{Model integration improves decoding of superior temporal and inferior frontal brain regions}

Thus far, analyses have been based on decoding the semantic network of all 22 ROIs as an ensemble. Mean \pm SEM decoding accuracies across participants for individual ROIs are in Figure 6. As displayed in Figure 6, decoding accuracies arising from the multimodal approach were significantly greater than those for either model in isolation in the left superior temporal sulcus 


\section{Fraction of variance explained}

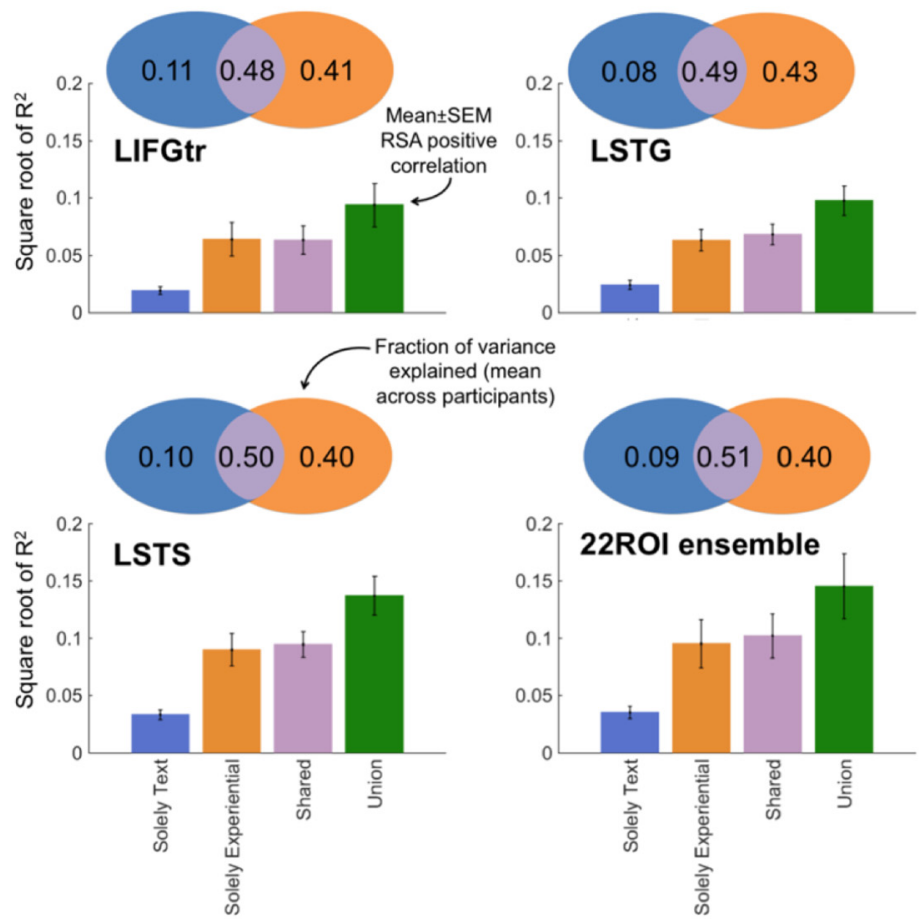

\section{Partial correlation}
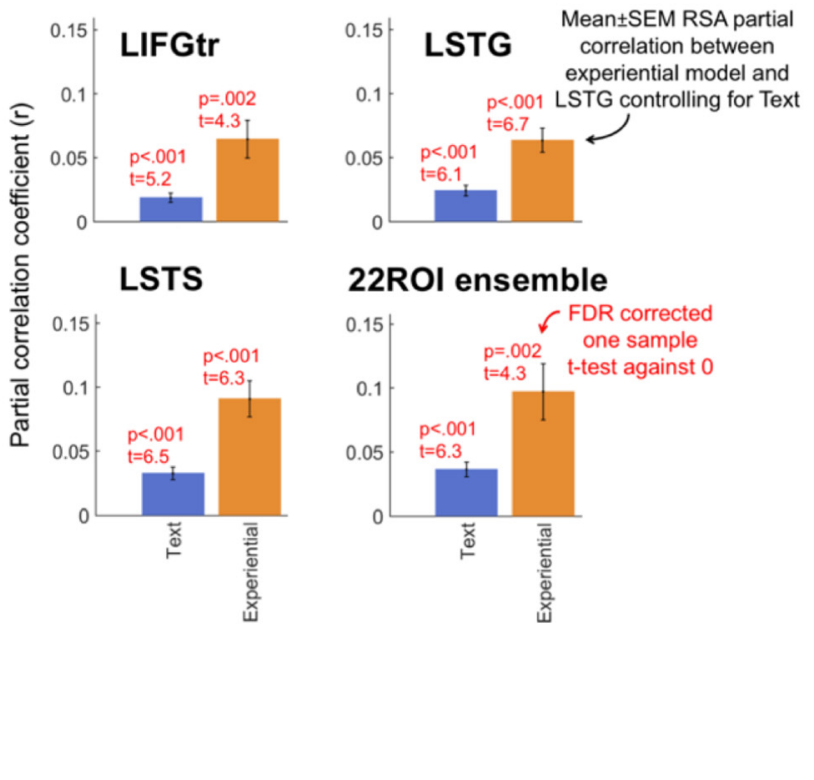

\section{ROI ensemble}

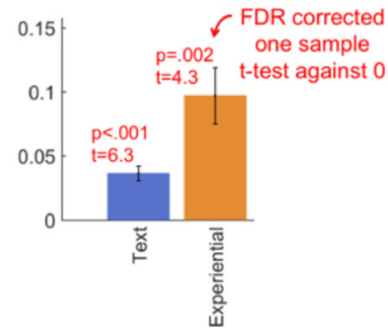

Figure 7. Partitioning the contribution made by the text-based and experiential models to explaining neural similarity structure across the entire set of 240 sentences. Left, Venn diagrams represent the mean (across participants) fraction of variance that is solely accounted for by the individual models and shared between them in the RSA analyses (see Fig. 3). Left, Bar plots represent the associated mean \pm SEM positive correlations (square root of $R^{2}$ ). Deserving of additional explanation, in LIFGtr, the mean experiential coefficient is marginally greater than the shared coefficient, whereas the mean fraction of variance explained by the experiential model is less than the shared component. This occurred because the experiential model tended to uniquely explain more variance in participants with large Union $R^{2}$ values (relative to shared variance) and vice versa. The averages of raw coefficients (in the bar plots) reflect this trend, but the Venn diagrams do not because the trend was removed by computing fractions within each participant, before averaging across participants. Mean \pm SEM partial correlation coefficients for the two models in the same RSA analyses are displayed in the four bar plots to the right (and tested against zero).

(LSTS), left superior temporal gyrus (LSTG), and the triangular part of the left inferior frontal gyrus (LIFGtr), and not reduced for any ROI. LSTS and LSTG yielded the highest and second highest decoding accuracies across all ROIs. All ROIs were on average decoded better than chance by both models. However, no statistically significant differences in overall decoding accuracy were detected between models for any ROI. Otherwise, decoding accuracies varied across ROIs in a similar fashion to that observed in previous results (Anderson et al., 2017a, 2019) and were strongest in LSTS with a mean accuracy of 0.75 (permutation tests revealed all individuals were decoded at accuracies significantly above chance level, $p=0.05$ ). The lowest average decoding accuracy was for the right angular gyrus, mean $=0.59$, with 7 of 14 participants returning results that were significantly above chance $(p=0.05)$.

\section{Post hoc tests partitioning the variance in the neural}

\section{similarity structure explained by each model}

The fraction of variance in the overall representational similarity structure (derived from all 240 sentences) of LSTS, LSTG, LIFGtr, and the 22 ROI ensemble that was uniquely explained by each model or commonly explained by both models is in the Venn diagrams of Figure 7 . In each of the four tests, $\sim 50 \%$ of the captured variance in neural similarity structure could be explained equally by either model (within-participant percentage averaged across participants). Approximately $40 \%$ of the remaining variance was associated with the experiential model, and the other $10 \%$ associated with the text-based model. Also displayed in Figure 7 (right) are partial correlation coefficients, reflecting the correlation between the neural sentence-level similarity structure, and the text-based model, while controlling for the experiential model (and vice versa). For all ROIs, partial correlation coefficients for both the text-based and experiential models were found to be significantly greater than zero (across participants, test statistics are in Fig. 7). This provides further evidence that both models independently contributed to explaining the neural data.

In both of the post hoc RSA analyses, the contribution of the experiential model is on face value stronger and the text-based model weaker than would have been anticipated from the previous decoding analyses in which accuracies arising from each individual model were fairly well balanced (Fig. 6). We are currently unsure of the precise reason for this. The decoding analysis differs from the current RSA in two key respects. First, it repeatedly tests different subsamples of the representational similarity matrix that correspond to particular sentence pairs. Specifically, each cross-validation iteration is based on a test of $238 \times$ 2 correlation coefficients rather than the global set of all 28,680 coefficients as tested by the RSA. Second, the decoding analysis incorporates a decision function to best match up model sentences with fMRI sentences. Thus, it seems that one, other, or both of these differences render the decoding analysis less sensitive to emphasizing the contribution of the experiential model. We leave detailed investigation of these differences over to future work. However, in the meantime, the current post hoc analyses 
Note: Cross-subject (brain activation-based) decoding is liable to decode semantic and non-semantic (e.g. orthographic/syntactic) elements of neural activation
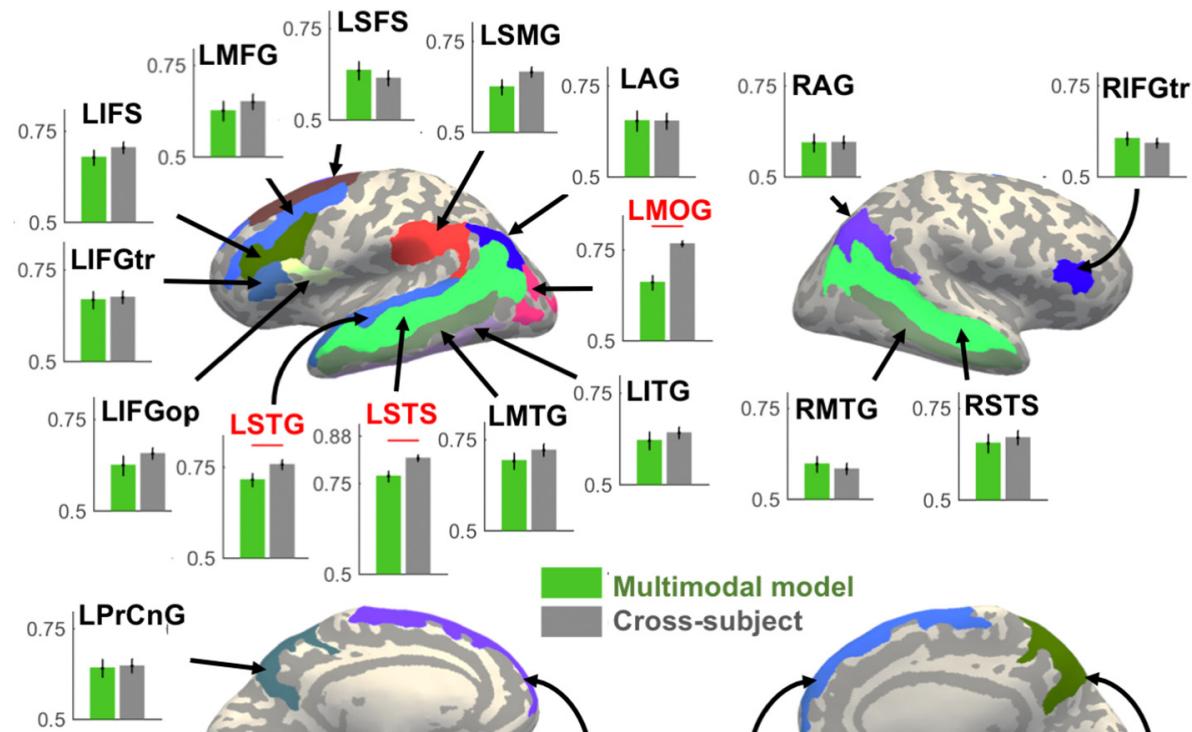

Multimodal model
Cross-subject
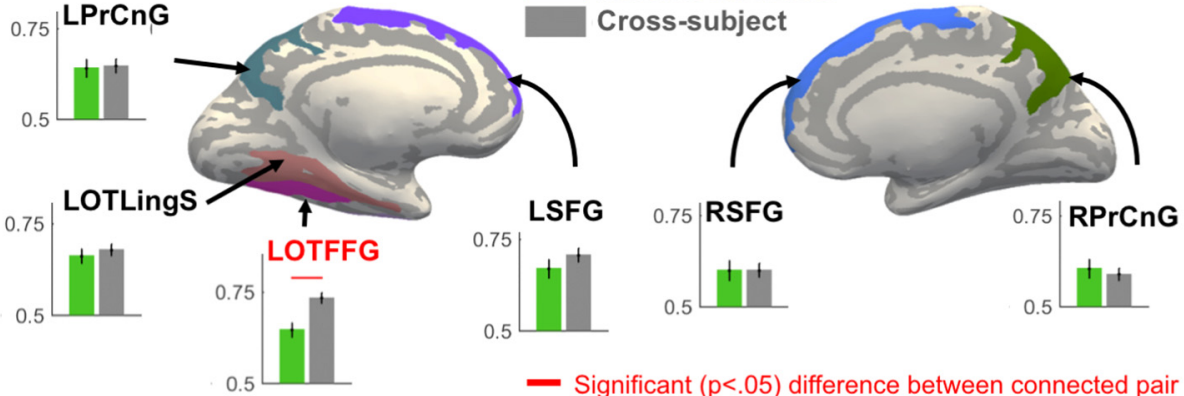

- Significant $(p<.05)$ difference between connected pair (paired t-test, FDR corrected)

\section{ROls decoded as an ensemble}

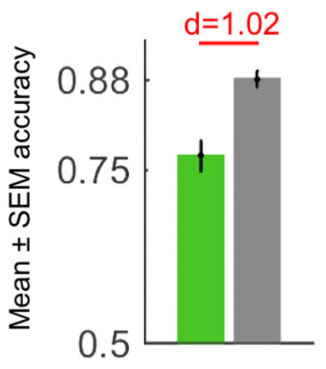

Cross-subject approach particularly improves on decoding of sentences containing abstract words

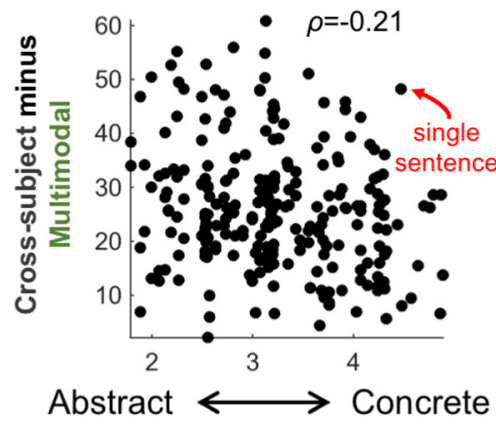

Concreteness rating of most abstract word in sentence (1 to 5)

Figure 8. Estimating the room for improvement: how cross-participant decoding improves on the multimodal model-based approach. Data are mean \pm SEM cross-participant (brain-based) decoding accuracies (see Fig. 2, bottom) across all 14 participants beside comparative results for the multimodal model (also shown in Fig. 4). Right, Detailed results arising from decoding using the combination of all $22 \mathrm{ROIs}$ (see Fig. 2, top). Scatter plots represent characteristics of sentences for which cross-participant (brain-based) decoding was advantaged over the multimodal model-based approach. The effect size ( $d$ ) was estimated as described in Figure 4. $\rho$ corresponds to Spearman's correlation coefficient.

underline the value of the experiential model in explaining the neural data.

Cross-participant neural decoding estimates an upper bound on decoding accuracy

Having demonstrated the benefits of combining models, we questioned how much room there is left over for improvement in decoding. We asserted that, in the general case, the best decoder of an individual's fMRI activation will be other people's fMRI activation (at least in the absence of a personalized semantic model). To this end, we decoded each individual's fMRI data using all other individuals in turn and then combined the collective decoding decisions together as an estimate for the upper bound on decoding accuracy. In advance, such cross-participant decoding is liable to be advantaged over the semantic models by additionally decoding nonsemantic information (e.g., activation reflecting orthographic or syntactic processing). Nevertheless, this information is still useful to identify ROIs for which there is room for improvement in decoding.

As illustrated in Figure 2 (bottom), we first used each participant to decode each other participant (repeated for each ROI, and the combination of 22 ROIs). For each individual, this left 13 decision matrices $(2 \times 2)$ at each cross-validation iteration (for each of the 13 other participants). These 13 matrices were pointwise averaged, scored as previously by comparing the sums on the matrix diagonal and antidiagonal, and then scores were averaged across all cross-validation iterations to give a final cross- participant decoding accuracy. We considered this final accuracy as an estimate on the upper bound decoding achievable for that individual (in absence of a personalized semantic model).

Mean \pm SEM cross-participant decoding accuracies for each ROI are compared with the multimodal decoding accuracies in Figure 8. Only multimodal decoding accuracies (i.e., the best decoder so far) are displayed as a comparison to avoid visual clutter (the isolated model results are in Fig. 6). FDR-corrected paired $t$ tests between cross-participant and multimodal modelbased accuracies revealed significantly stronger decoding for the cross-participant approach in LSTS, LSTG, left occipitotemporal fusiform gyrus (LOTFFG), and left mid occipital gyrus (LMOG) and for the set of 22 ROIs decoded as an ensemble (all $p<0.05$ ). This indicated that there was sentence-related signal present in the fMRI data within these regions that had not been decoded by the models. This was particularly the case for LMOG and LOTFFG ( $d=1.67$ and 1.24 , respectively); however, improvements for LSTS and LSTG were notable $(d=0.81$ and 0.56 , respectively).

Also noteworthy was the gap separating cross-participant decoding accuracies for the 22 ROI ensemble and individual ROIs. For instance, the difference between cross-participant decoding of the highest accuracy ROI (LSTS) and the 22 ROI ensemble was significant and sizeable $(t=7.7, p<0.0001, d=1.47)$. This provides evidence that complementary sentence-related information (which could be semantic or orthographic or syntactic) was distributed across the different ROIs. In contrast for the se- 
mantic model-based analysis, most of the decodable information appears to be in LSTS (see also Figs. 9-11).

\section{Sentences decoded weakly by the models tended to contain abstract words}

To attempt to get a handle on what semantic information could have been left undecoded by the models, and in so doing identify model weaknesses we finally tested whether particular types of sentences were better decoded by the cross-participant approach. We concentrated analyses on decoding all 22 ROIs as an ensemble. Then for each participant (14), we extracted sentence-wise decoding scores (240) for both the cross-participant and multimodal approaches. The vector of 240 multimodal model-based decoding scores was pointwise subtracted from the crossparticipant decoding scores to generate an "accuracy boost vector" indicating which sentences were better decoded by cross-participant decoding. For each participant, we correlated (Spearman) this accuracy boost vector with $\mathrm{min} / \mathrm{mean} / \mathrm{max}$ concreteness of constituent content words in sentences, the $\mathrm{min} /$ mean/max ( $\log 2$ transformed) word frequency per sentence, the minimum/mean/maximum word length per sentence, and the number of words per sentence. The resulting correlation coefficients were Fisher's $r$-to- $z$-transformed (arctanh). For each set of 10 tests, transformed coefficients for all 14 participants were compared with zero using a $t$ test. $p$ values were then FDRcorrected. Four tests yielded significant results. These were the concreteness rating of the most abstract word in the sentence (mean $r=-0.08, t=-4.1, p=0.01$ FDR-corrected; see also Fig. 8), mean word frequency (mean $r=-0.06, t=3.6, p=0.03$ FDR-corrected), minimum word length (mean $r=-0.05, t=$ $-3.5, p=0.03$ FDR-corrected), and the number of words in the sentence (mean $r=0.07, t=4.5, p=0.01$, FDR-corrected). To counteract possible effects of spurious intercorrelations between these four sentence measures, a second round of partial correlation analyses was run. Each participant's accuracy boost vectors were partially correlated (Spearman) with vectors associated with each of the four measures in turn while controlling for the other three. Of the four measures, only correlations with the concreteness rating of the most abstract word in the sentence were found to be significantly lower than zero (mean partial $r=-0.06, t=$ $-3.6, p=0.003$ ). This provided evidence that cross-participant decoding gained a particular advantage over the multimodal model approach for sentences containing abstract words. The relationship between the decoding advantage and the number of words per sentence, constituent word frequencies, and word lengths is unclear due to their intercorrelation.

\section{Secondary supporting analyses}

The following three sections present the outcome of a series of secondary supporting analyses that are possibly best suited to the dedicated reader.

\section{Supporting analysis: multimodal decoding advantage is preserved in analyses of different cortical networks}

Our main analysis was focused on a semantic network of 22 regions that had been identified by Anderson et al. (2019), which differently used a two-stage regression-based analysis and the experiential model alone. Reasons for this were to maintain continuity, so as to enable current results to be referenced back to the results of Anderson et al. (2019), and also for simplicity. However, because the network of 22 regions was derived using the experiential model only, it is possible that the current analysis could have been biased toward the experiential model.
To confirm that results were not specific to the 22 ROI network, we repeated our initial decoding analysis using different network configurations. We first derived decoding accuracies for all 150 ROIs in the Destrieux atlas using the text-based and experiential models independently. At this stage, the analysis was conducted without voxel selection to cut down on computational overheads. We then identified a set of "high accuracy" brain regions that were decoded significantly at the $p=0.01$ level (FDRcorrected) by either model. We then reperformed the decoding analysis, this time with voxel selection (50 voxels per ROI). We first decoded the union of high accuracy ROIs associated with either or both of the models. Second, we decoded the intersection of high accuracy ROIs associated with both models.

The "intersection" network contained 12 ROIs. Of these, the following 9 of 12 ROIs also appeared in the 22 ROI ensemble: LSTS, LSTG, LMTG, LIFGtr, LIFS, LAG, LPrCnG, LOTLingS, and RSTS. Destrieux atlas names for the 3 ROIs that were not in the 22 were as follows: ctx_lh_G_cingul-Post-dorsal, ctx_lh_ S_oc-temp_lat, ctx_lh_S_subparietal. The "union" network contained 15 ROIs. These included the 12 listed above as well as LOTFFG and LMOG, which were in the original 22 ROI ensemble, and ctx_lh_S_precentral-inf-part, which was not. The 4 new ROIs that had not been in the original 22 were all relatively low scoring (ranked 11th highest or below out of the union of 15 ROIs). Mean \pm SEM decoding accuracies for the text-based, experiential, and multimodal for the 4 new ROIs were, respectively, as follows: ctx_lh_G_cingul-Post-dorsal $0.63 \pm 0.02,63 \pm 0.02$, $64 \pm 0.02$; ctx_lh_S_oc-temp_lat $0.63 \pm 0.02,63 \pm 0.01,65 \pm$ 0.02; ctx_lh_S_subparietal: $0.62 \pm 0.02,63 \pm 0.02,64 \pm 0.02$ and ctx_lh_S_precentral-inf-part $0.62 \pm 0.02,61 \pm 0.02,63 \pm 0.02 . t$ tests revealed no significant differences in decoding accuracy between the text-based and experiential models for these 4 ROIs.

Decoding accuracies arising from the intersection and union networks are displayed in Figure 9. For comparison, we display results of the original $22 \mathrm{ROI}$ ensemble analysis conducted on either 50 or 100 voxels selected per region. Additionally, we display results for LSTS (the highest scoring ROI) and also results when the analysis was undertaken on the entire cortex without any voxel selection. Also displayed are correlations relating the contribution of the text-based model to multimodal decoding to ratings of sentence abstractness echoing Figure 3 .

Figure 9 reveals that the decoding advantage brought through model integration is preserved across all tests regardless of the network configuration. Also preserved is the "abstractness" advantage brought by the text-based model to decoding sentences containing abstract words. It is visually apparent that that decoding accuracy was modulated by the network configuration tested. Because it is not a focus of the current article, we leave in-depth treatment of these differences and how to select the optimal network over to future work. Suffice it to say that activity in LSTS appears to have been the linchpin of network-based decoding, and there was no dominant network configuration that yielded significantly greater accuracy than all others.

\section{Supporting analysis: comparison and integration of ridge regression with similarity-based decoding}

Prompted by a concern that the current RSA approach might inherently bias results in favor of one or other model, we used ridge regression (Hoerl and Kennard, 1970) to reanalyze multimodal ROIs: LSTS, LSTG, LIFGtr, and the 22 ROI ensemble. Ridge regression was selected because it has been popular in recent fMRI studies using text-based models (e.g., Huth et al., 


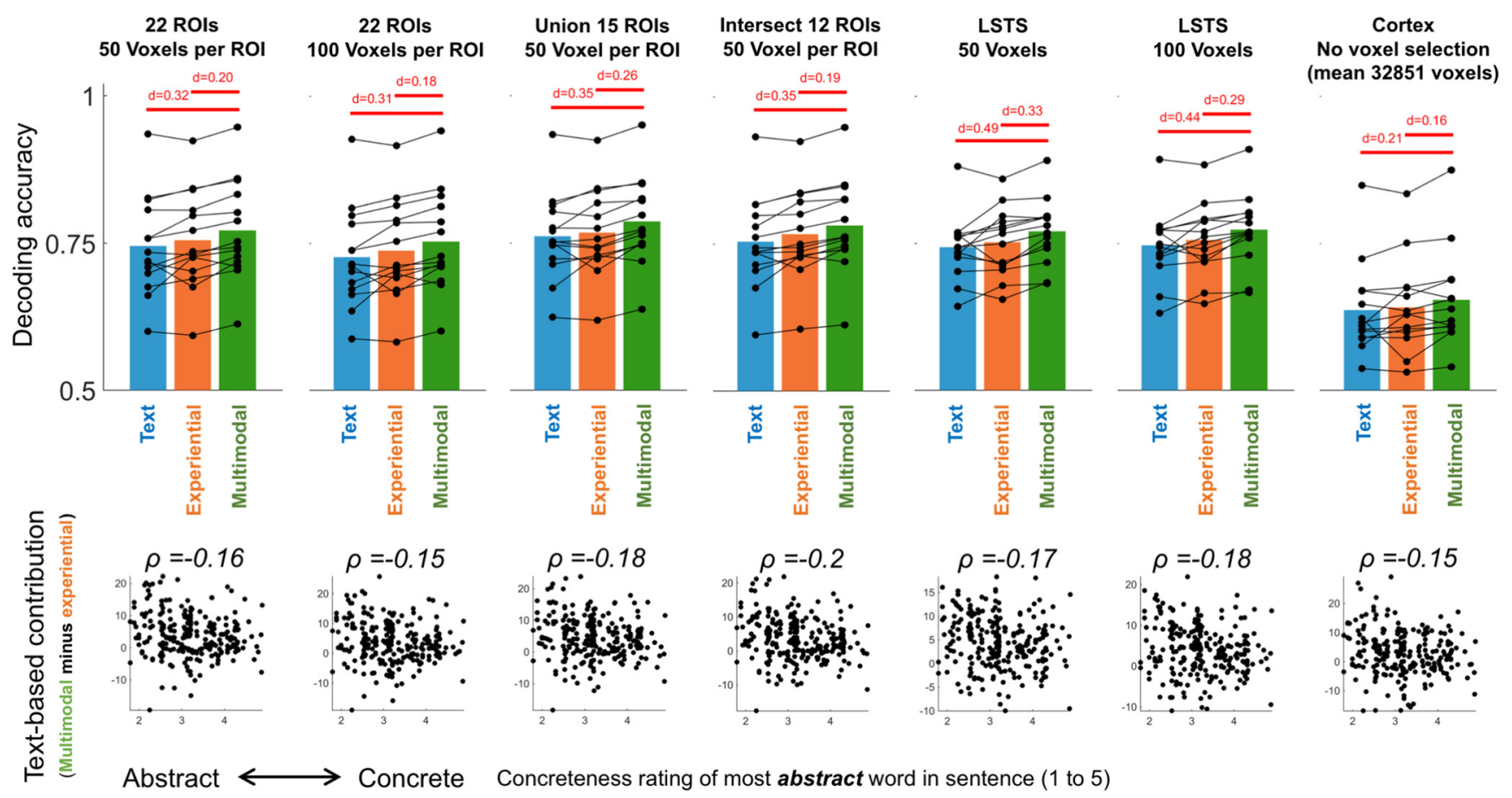

Figure 9. Decoding accuracies arising from decoding different networks of ROls using different model combinations. This figure is a companion to Figure 4, which describes how effect sizes (d) were estimated. $\rho$ corresponds to Spearman's correlation coefficient.

2016a; de Heer et al., 2017; Pereira et al., 2018). Importantly, this reanalysis provides a quantitative estimate of the decoding advantages brought by using experiential attributes and similaritybased methods comparative to a state-of-the-art text-based/ridge regression approach.

For each participant and each ROI, we reran precisely the same leave-2-sentence-out cross-validation procedure with the same training/testing data splits and same voxel selection (50 voxels per ROI) as our main similarity-based analysis. At each of the 28,680 cross-validation iterations, we fit a separate ridge regression for the text-based model and then for the experiential model to predict activation in each individual voxel (i.e., forward encoding). We repeated regression fitting using each of the following 9 regularization penalties $(\lambda)\left(1,10,100,10^{3}, 10^{4}, 10^{5}\right.$, $10^{6}, 10^{7}$, and $\left.10^{8}\right)$. As before, at each cross-validation iteration, we computed a $2 \times 2$ decoding decision matrix for both the text-based and experiential models. This was repeated for each $\lambda$ by correlating predicted and actual fMRI activation patterns and $r$-to- $z$-transforming correlation coefficients ( 9 decoding decision matrices per model). To create a multimodal $2 \times 2$ decoding decision matrix, we pointwise averaged together decision matrices arising from each of the two models precisely as described in Figure 1 (Stage 4). We repeated this for every combination of $\lambda$ pairs, leaving $9 \times 9=81$ multimodal decision matrices per iteration per ROI. We created a 22 ROI ensemble decoding matrix for each model and $\lambda$ combination by pointwise averaging each of the $9+9+81$ decision matrices across ROIs. For the multimodal approach, there were $81^{22}$ possible ways that $\lambda$ could have been combined across ROIs; therefore, the pointwise averaging approach we have taken could have missed out on the ideal combination. Alternative approaches could have included selecting an optimal $\lambda$ for each ROI using nested cross-validation, or building a single decision matrix from all voxels. Because regression is not our prime focus, we leave detailed investigation of this to future work. As for all our other analyses, at each iteration, decoding decision matrices were evaluated as correct (1) if the sum of coefficients on the diagonal exceeded the sum on the antidiagonal; otherwise, they were incorrect (0). A final decoding accuracy score was assigned as the mean correctness across trials for each model and $\lambda$ combination (yielding $9+9+81$ accuracies per ROI per participant).

Decoding accuracies were in general qualitatively similar to those observed for the similarity-based analysis and are illustrated in Figure 10. Decoding accuracies for both models tended to reach a maxima and flatten off at $\lambda$ values greater than $\lambda=10^{3}$ or $10^{4}$. For "flattened" $\lambda$ values, in tests on LSTS and LIFGtr, the multimodal approach was significantly more accurate than either of the text-based and experiential models alone. For LSTG and the 22 ROI ensemble, whereas the multimodal approach yielded significantly greater decoding accuracies than the text-based model (Fig. 10, bottom left), accuracies were not significantly greater than the experiential model (at least for "flattened" $\lambda$ values Fig. 10, bottom right). This weak multimodal improvement reflects both the comparatively weak decoding accuracies achieved for the text-based model using ridge regression, and the relatively strong accuracies for the experiential model (for statistical test results, see Fig. 10, legend). Ridge regression's weak performance with the text-based model could reflect a combination of difficulties surrounding the following: scaling up to the 300 text-based features (compared with 65 experiential attributes, with 240 sentences); and/or the distributional properties of the text-based data; and/or our current selection of $\lambda$ missing out on the optimal value. Ridge regression's stronger performance with the experiential model evidences that parameter fitting can lead to decoding improvements with the current data. There was little to separate multimodal decoding accuracies for the two decoders; although for a particular $\lambda$ value $\left(10^{3}\right)$, ridge regression yielded stronger performance in LSTS alone.

The comparatively weak decoding accuracies obtained with ridge regression for the text-based model begged the question of 


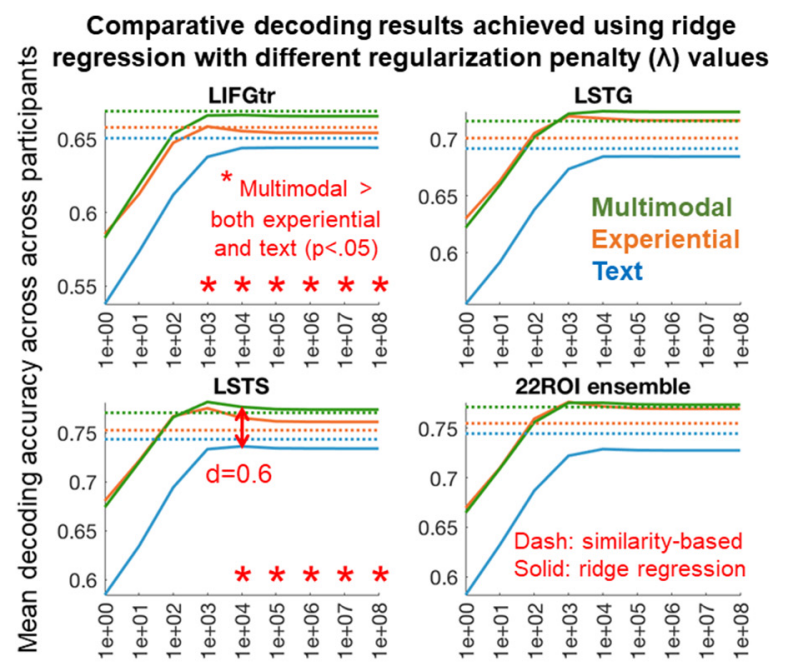

$\lambda$ (same value for both text and experiential models which corresponds to the matrix diagonal of the other plots)

T-tests between text and multimodal modelbased decoding accuracy for different $\lambda$ pairs

LIFGtr

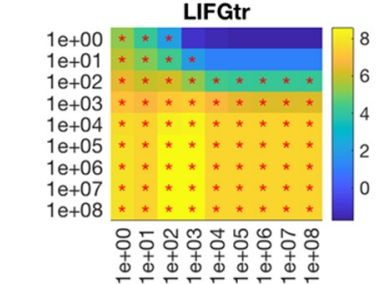

LSTS

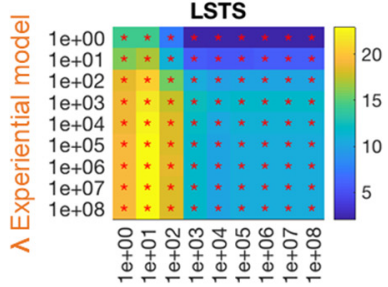

$\lambda$ Text model

(same for uni/multimodal)

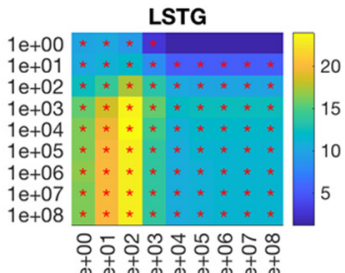

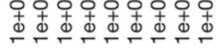
22ROI ensemble

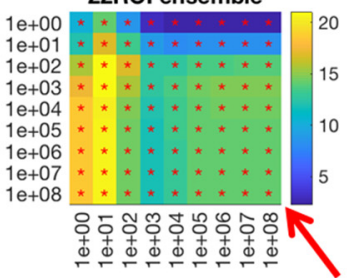

Stars in every cell indicate multimodal decoding significantly $(p<.05)$ improved over the text model for all $\lambda$ pairs tested

Multimodal model-based decoding accuracy for different ridge regularization penalty $(\lambda)$ pairs

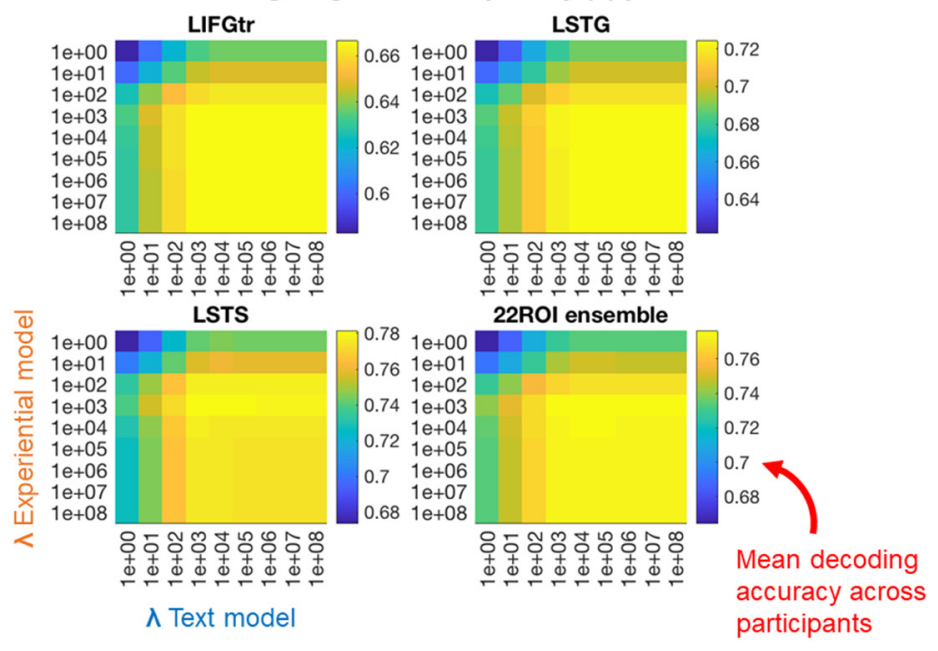

T-tests between experiential and multimodal modelbased decoding accuracy for different $\lambda$ pairs

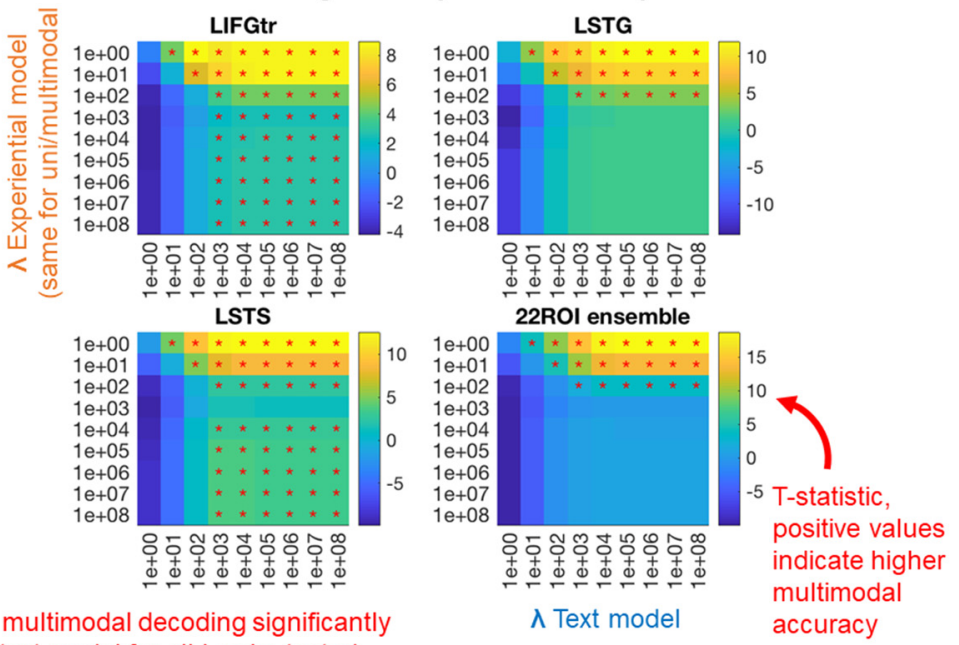

Figure 10. Comparative text-based, experiential, and multimodal decoding accuracies acquired using ridge regression. Top, Left, Multimodal advantages for a particular selection of $\lambda$ values. Top, Right, Multimodal decoding accuracies for all $\lambda$ configurations. Results of paired $t$ tests comparing multimodal decoding accuracies with text-based decoding accuracies (bottom, left) and experiential decoding accuracies (bottom, right) for each $\lambda$ configuration. All tests were one-tailed, in anticipation of the multimodal advantage observed in our initial analyses. The illustrated effect size (d) provides a conservative estimate of the benefit of integrating experiential features into a conventional text-based ridge regression approach. $d$ was computed as described in Figure 4 (legend). Differences between ridge regression and similarity-based decoding: Top, Left, Both similarity-based (dashed lines) and regression-based (solid lines) results. Decoding accuracies using ridge regression with the text model and the top performing $\lambda\left(\right.$ always $\lambda=10^{4}$ ) were unanimously significantly lower than for the similarity-based approach $\left(\right.$ LSTS: $t=5.8, p=6 \times 10^{-5}, \mathrm{df}=$ 13; LSTG: $t=5.3, p=1.4 \times 10^{-4}, \mathrm{df}=13$; LIFGtr: $t=5.2, p=1.8 \times 10^{-4}, \mathrm{df}=13 ; 22 \mathrm{ROI}: t=8, p=2 \times 10^{-6}, \mathrm{df}=13$; all two-tailed paired $t$ tests, $\left.\mathrm{df}=13\right)$. Conversely, in $75 \%$ of tests using the experiential model with the top scoring $\lambda$ (always $\lambda=10^{3}$ ), ridge regression yielded significantly stronger decoding accuracies than the similarity-based analysis (LSTS: $t=5.6, p=$ $8.5 \times 10^{-5} ;$ LSTG: $t=4, p=0.001$; LIFGtr: $t=0.1, p=0.9 ; 22 \mathrm{ROI}: t=7, p=9.8 \times 10^{-6}$; all two-tailed paired $t$ tests, $\left.\mathrm{df}=13\right)$. For multimodal decoding, ridge regression yielded stronger decoding in LSTS with the top scoring $\lambda=10^{3}(t=3.3, p=0.006)$ but not other $\lambda$ values; otherwise, there were no significant differences for the other ROIs (LSTG: $t=1.9, p=0.07$; LIFGtr: $t=$ $-0.88, p=0.4 ; 22 \mathrm{ROI}: t=1.4, p=0.2$; all two-tailed paired $t$ tests, $\mathrm{df}=13$ ). All $p$ values are not corrected for multiple comparisons.

whether the ridge regression multimodal decoder was failing to capitalize on neural information that was decoded successfully by the similarity-based approach. Relatedly, whether decoding of LSTG and the 22 ROI ensemble would be advantaged by a "best of both worlds" multimodal approach that jointly leverages similarity-based decoding and ridge regression. To answer this question, we reran the cross-validation analysis using the similarity-based approach with the text-based model in parallel with ridge regression on the experiential model (using top scoring $\lambda=10^{3}$ ). At each cross-validation iteration, we integrated the respective decoding decisions made by the different models/decoders by pointwise summing respective $2 \times 2$ decoding decision matrices (as in Fig. 1, Stage 4). The multimodal joint similarity/ regression approach indeed yielded significantly greater decoding accuracies than both the regression-based experiential decoder and the text similarity-based decoder in LSTG and the 22 ROI ensemble as well as LSTS and LIFGtr (for statistical test results, see Fig. 11, legend).

In sum, this section has provided further evidence that experiential semantic features explain variance in sentence-level fMRI data that cannot be accounted for by state-of-the-art text-based regression approaches, and further support for the claim that multimodal approaches provide the most accurate models of fMRI to date. 
Text-based decoding using similarity approach

Experiential attribute decoding using ridge regression

Multimodal decoding using a fusion of the above
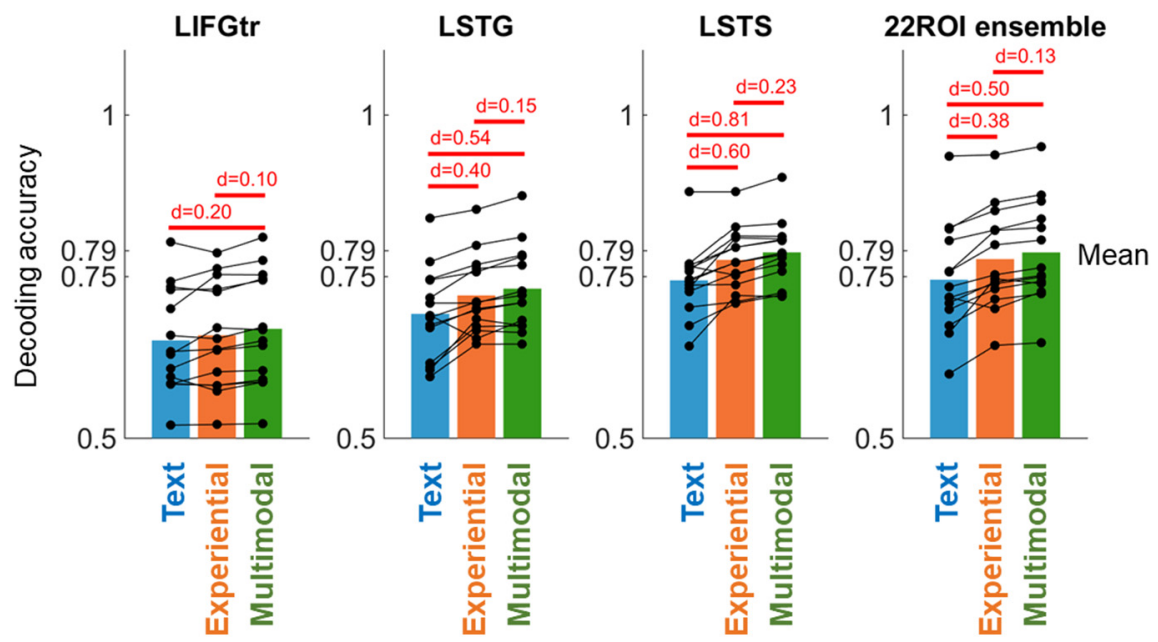

Figure 11. Multimodal decoder integrating the best text-based decoder (similarity) with the best experiential decoder (ridge regression, $\lambda=10^{3}$ ). Effect sizes ( $d$ ) are displayed in cases of statistically significant differences (paired $t$ test, all $p \leq 0.01$, FDR-corrected). $d$ was computed as described in Figure 4. Paired $t$ test results were as follows: for contrasts between multimodal decoding and experiential regression-based decoding: LSTS: $t=4.8, p=0.002$; LSTG: $t=3.6, p=0.01$; LIFGtr: $t=4.5, p=$ $0.003 ; 22 \mathrm{ROI}: t=5, p=0.002$; for contrasts between multimodal decoding and text similarity-based decoding: LSTS: $t=8.3$, $p<10^{-4}$; LSTG: $t=8.0, p<10^{-4}$; LIFGtr: $t=4.0, p=0.006 ; 22 \mathrm{ROI}: t=5, p<10^{-4}$; for contrasts between experiential regression-based decoding and text similarity-based decoding: LSTS: $t=4.2, p<0.005$; LSTG: $t=3.8, p<0.009$; LIFGtr: $t=$ $1.39, p=0.58 ; 22$ R0l: $t=4.8, p=0.002$. All $p$ values FDR-corrected.

\section{Supporting analysis: persistence of multimodal advantage using different text-based models}

The claim that the experiential model enhanced decoding by capturing nonlinguistic experiential knowledge rests on the assumption that the current text-based model captured all of the experiential structure that is possible to obtain from word use statistics (see Materials and Methods). As both the text-based and experiential modeling approaches are in an ongoing state of development, it cannot be concluded that the current results will be the same for all future models and/or neural datasets. Additionally, it is possible that idiosyncrasies surrounding how the experiential model was constructed and its statistical properties (e.g., representational sparseness) could have contributed to the decoding advantage it conferred. Vice versa for the text-based model. Consequently, we consider that the current results provide "early evidence" that linguistic and nonlinguistically acquired knowledge is represented in fMRI activation elicited in sentence comprehension. However, our core finding that an integrated experiential and text-based decoding approach yields significantly higher accuracy than either model alone has held true for all text-based models we have tested thus far, which have been built using different algorithms from different text corpora. We are not aware of another experiential model suitable for the current analysis.

In preliminary investigations, we had tested word cooccurrence models (e.g., Roller et al., 2014), word2vec (Mikolov et al., 2013; Baroni et al., 2014), which yielded similar decoding accuracy levels (e.g., LSTS: mean $\leq 0.75$ ), and a similar core result that integrating experiential and text-based models yielded significantly greater decoding accuracies (e.g., LSTS: Multimodal $>$ co-occurrence $t=10.1, p<10^{-5}, d=0.78$; Multimodal $>$ word2vec $t=6.9, p<10^{-5}, d=0.44$, both two- tailed paired $t$ tests). We focused on GloVe (Pennington et al., 2014) principally because it was the basis for Pereira et al. (2018) fMRI sentence decoding study.

In the interim, a number of new computational text-based approaches have emerged (e.g., Conneau et al., 2017; Peters et al., 2018; Subramanian et al., 2018; Devlin et al., 2019). These typically leverage deep artificial neural networks to derive sentence representations that reflect word order and within-sentence contexts. A thorough comparison of deep network text-based approaches and the experiential model is beyond the scope of the current work, and perhaps would be best undertaken using an experiential model that also accommodates word order and context effects (which could be achieved by rating entire sentences, words in context, or entering experiential word vectors as deep network input). Nevertheless, to date, we have tested one deep model, InferSent (Conneau et al., 2017), which is notable in having recently yielded state-of-the-art decoding of Pereira et al. (2018) sentence-level fMRI dataset (Sun et al., 2019). We hope to present a full treatment of results in future work, however, to foreshadow those, the current core finding still holds: Integrating the current sentence-level experiential model with InferSent yields significantly greater decoding accuracy than either model in isolation (e.g., LSTS: Multimodal $>$ InferSent $t=7.2, p<10^{-5}, d=0.34$, two-tailed paired $t$ test).

\section{Discussion}

This study has revealed early evidence that modeling both linguistic knowledge of word usage and experiential knowledge of words' referents enhances decoding of brain activation patterns associated with sentence meaning. This suggests that nonlinguistic experiential knowledge is represented in sentence-level fMRI activation. Importantly, because this result is based on direct measures of brain activation elicited during the comprehension of natural sentences, it is an advance on previous behavioral evidence that has been indirectly inferred from experimental responses (e.g., Paivio, 1971; Stanfield and Zwaan, 2001; Zwaan et al., 2002; Andrews et al., 2009; Louwerse and Jeuniaux, 2010; Kousta et al., 2011). More generally, this is evidence that it is now possible to use brain data to quantitatively estimate the contribution that linguistic and nonlinguistically acquired knowledge makes to representing the meaning of natural language. This is especially relevant to theories that conceptual representations are acquired through and partially embodied within experiential neural systems (Barsalou et al., 2008; Glenberg, 2010; Pulvermüller, 2013; Binder et al., 2016). Relatedly, it suggests that we can begin to estimate how close "ungrounded" semantic models (e.g., text-based) can get to representing human conceptual knowledge (for discussion of the "symbol grounding problem," see Harnad, 1990). With respect to questions of grounding and embodiment, we should be clear that the current analyses provide no guarantee that brain activation that was selectively decoded by the experiential model was 
actually represented within primary perceptual/modal processing systems or was critical to comprehension rather than epiphenomenal (Mahon and Caramazza, 2008; Mahon, 2015).

At a more practical level, the current study advances on previous state-of-the-art text-based neural encoders/decoders (Huth et al., 2016a,b; Pereira et al., 2018) because multimodal integration boosts decoding performance. This provides further evidence that combining multiple modalities of information in semantic models leads to more human-like representations of meaning (Andrews et al., 2009; Bruni et al., 2014; Anderson et al., 2015).

We have also demonstrated that the text-based model contributed particularly to decoding sentences containing abstract words. Although this was hypothesized (Anderson et al., 2017a) and text-based models have previously helped explain abstract concept fMRI (Anderson et al., 2017b; Wang et al., 2018; Pereira et al., 2018), it was not a foregone conclusion. This was because abstract concepts are thought to be grounded relatively strongly upon affective experiences (Kousta et al., 2011; Vigliocco et al., 2014) and contemporary text-based models generate relatively weak predictions of affective experiential attributes (Utsumi, 2018). As it turned out, many sentences that the text-based model helped explain contained words with affective connotations (e.g., "happy," "celebrated," "survived"). It seems likely that the advantage conferred in these cases (and others) was down to the extra linguistic/contextual information in the text-based model. Otherwise, Utsumi (2018) found text-based models to be disadvantaged in predicting spatial/temporal attributes. Testing how spatial/temporal attributes contribute to semantic representations in the brain may thus provide an interesting avenue for future investigation. While we here detected tentative evidence that the experiential model contributed to decoding concrete sentences (without any abstract words), this result did not survive correction for multiple comparisons.

The demonstration that sentence-level neural activation is best decoded using a multimodal approach is not without foreshadow. Anderson et al. (2015) found that a visually grounded semantic model (derived from natural images) and a text-based model differentially correlated with fMRI activation in brain regions with known visual/linguistic processing roles. However, because participants were tasked to read concrete nouns and actively contemplate their semantic properties (Just et al., 2010), it is not clear whether the results reflect active visual imagery as opposed to more passive language comprehension (see also Willems et al., 2010). In other work, Abnar et al. (2018) used a joint text-based/experiential approach to better predict fMRI elicited by drawings of nouns alongside their names, and Wang et al. (2018) revealed partial correlations between fMRI elicited by abstract Chinese words, a Chinese text-based model, and a model built from 12 behavioral ratings that interestingly included valence, space, and time. In both cases, it is not clear how results would extend to decoding read sentences (in English).

The current study extended a representational similaritybased decoding method (Anderson et al., 2016, 2017b) to the neural decoding of sentences using parallelized combinations of multiple models, brain regions, and participants. Combination of multiple participants' neural data was achieved by "ensemble averaging" of decoding decisions. This sets the similarity-decoding method apart from "hyper-alignment" methods (Haxby et al., 2011; Guntupalli et al., 2016) that represent neural responses using a common representational space. A disadvantage of integrating decoding decisions is that this does not generate predictions of individual voxel's activity (unlike regression-based encoding). Similarity-based approaches can be configured to estimate voxel activity by applying the correlation coefficients comprising similarity vectors (e.g., Fig. 1, Stage 3) as weights in a weighted average of corresponding brain activation patterns as described by Anderson et al. (2016). However, we leave a comparative investigation of this over to future work. We did not run the current analysis using similarity-based encoding in part to avoid the additional data normalization step that would have been required to combine data (Fig. 1, legend). For the decoding case at hand, the similarity-based approach performs competitively with ridge regression (better for the text-based model and worse for the experiential model; Fig. 10) while cutting out overheads associated with repeating the analyses with different regularization penalties, and picking the appropriate one.

Cross-participant neural decoding was introduced as a method to estimate an upper bound on decoding accuracy achievable with (group-level) models. This followed the reasoning that, on average, the most accurate neural decoder will be based on neural data. Indeed, for LSTS, LSTG, LOTFFG, and LMOG, decoding accuracy was significantly greater for the crossparticipant approach, and there were no ROIs for which accuracy was significantly worse. Practically speaking, this result was, however, not guaranteed. Had the fMRI data been too noisy and the group been too small, the model-based approach could have yielded stronger decoding. It is also important to recall that the upper bound estimate provided by cross-participant decoding does not apply to decoding semantics per se but to decoding the entire linguistic processing stream from stimulus perception to semantic interpretation. Also, the cross-participant approach does not apply to decoding person-specific aspects of semantic representation, so there may well be decodable neural signal left over that could only be revealed by personal information.

The upper bound decoding estimate was used to identify that weakly decoded sentences tended to contain abstract words, which suggests that the neural data contains undecoded aspects of abstract conceptual representations. This presents a challenge for future modeling to improve on models of abstract concepts. Given limitations in current understanding of abstract knowledge representation there may be an interesting opportunity to move forward here in a different way, by incorporating features of brain activation into artificial semantic models and in so doing provide a new way for neuroscience to feed back to AI (see also Fyshe et al., 2014; Hassabis et al., 2017).

One limitation of the current study is the assumption that the additional neural activation decoded by the experiential model reflects semantic information that cannot be extracted from natural language data. This is not strictly guaranteed, and it is possible that future text-based approaches will account for the signal decoded by the experiential model. A limitation of the current experiential approach is the assumption that experiential knowledge can be comprehensively estimated through introspective ratings of the relationship between concepts and putative neural systems. Indeed, we have revealed evidence that the text-based language model captures information the experiential model did not; however, there may be other semantic features that cannot be verbally described and/or introspectively accessed, in which case models that are truly grounded in modal information (e.g., Bruni et al., 2014; Anderson et al., 2015) may come to the fore. Ultimately, the answers to the above questions will be borne out through future work that incorporates different modalities of information into semantic models (e.g., Andrews et al., 2009; Bruni et al., 2014; Kiela and Clark, 2017), and compares this with brain data (e.g., Anderson et al., 2013, 2015, 2017b; Bulat et al., 
2017). The current study has contributed methods that we hope will assist in this enterprise.

In conclusion, the current study has provided initial evidence that linguistic and nonlinguistic experiential knowledge can be detected in sentence-level brain activation by extending a similarity-based framework to exploit respective models in fMRI decoding. It has also presented a cross-participant decoding method, which has demonstrated that a substantial amount of neural signal remains unexplained. This decoding gap is likely to be filled by modeling advances that take word order, syntax, morphology, and polysemy into account in semantic composition and begin to accommodate pragmatic inferences and theory of mind. For the future, in all of these endeavors, we contend that model-based approaches that integrate information across multiple modalities of experience will be necessary for the fullest interpretation of neural activation patterns associated with meaning.

\section{References}

Abnar S, Ahmed R, Mijnheer M, Zuidema W (2018) Experiential, distributional and dependency-based word embeddings have complementary roles in decoding brain activity. Proceedings of the 8th Workshop on Cognitive Modeling and Computational Linguistics (CMCL 2018), pp 57-66. Salt Lake City: Association for Computational Linguistics.

Anderson AJ, Lin F (2019) How pattern information analyses of semantic brain activity elicited in language comprehension could contribute to the early identification of Alzheimer's disease. Neuroimage Clin 22:101788.

Anderson AJ, Bruni E, Bordignon U, Poesio M, Baroni M (2013) Of words, eyes and brains: correlating image-based distributional semantic models with neural representations of concepts. Proceedings of the Conference on Empirical Methods in Natural Language Processing (EMNLP 2013), pp 1960-1970. Seattle: Association for Computational Linguistics.

Anderson AJ, Bruni E, Lopopolo A, Poesio M, Baroni M (2015) Reading visually embodied meaning from the brain: visually grounded computational models decode visual-object mental imagery induced by written text. Neuroimage 120:309-322.

Anderson AJ, Zinszer BD, Raizada RD (2016) Representational similarity encoding for fMRI: pattern-based synthesis to predict brain activity using stimulus-model-similarities. Neuroimage 128:44-53.

Anderson AJ, Binder JR, Fernandino L, Humphries CJ, Conant LL, Aguilar M, Wang X, Doko D, Raizada RD (2017a) Predicting neural activity patterns associated with sentences using a neurobiologically motivated model of semantic representation. Cereb Cortex 27:4379-4395.

Anderson AJ, Kiela D, Clark S, Poesio M (2017b) Visually grounded and textual semantic models differentially decode brain activity associated with concrete and abstract nouns. Trans Assoc Comput Linguistics 5:1730.

Anderson AJ, Lalor EC, Lin F, Binder JR, Fernandino L, Humphries CJ, Conant LL, Raizada RD, Grimm S, Wang X (2019) Multiple regions of a cortical network commonly encode the meaning of words in multiple grammatical positions of read sentences. Cereb Cortex 29:23962411.

Andrews M, Vigliocco G, Vinson D (2009) Integrating experiential and distributional data to learn semantic representations. Psychol. Rev 116:463498.

Andrews M, Frank S, Vigliocco G (2014) Reconciling embodied and distributional accounts of meaning in language. Top Cogn Sci 6:359-370.

Baroni M, Dinu G, Kruszewski G (2014) Don't count, predict! A systematic comparison of context-counting vs. context-predicting semantic vectors. Proceedings of the 52nd Annual Meeting of the Association for Computational Linguistics (Volume 1: Long Papers), Baltimore, Maryland, USA (2014). pp 238-247.

Barsalou LW (1999) Perceptual symbol systems. Behav Brain Sci 22:637660.

Barsalou LW, Santos A, Simmons WK, Wilson CD (2008) Language and simulation in conceptual processing. In: Symbols, embodiment, and meaning (De Vega M, Glenberg AM, Graesser AC, eds), pp 245-283. Oxford: Oxford UP.
Benjamini Y, Yekutieli D (2001) The control of the false discovery rate in multiple testing under dependency. Ann Statist 29:1165-1188.

Binder JR, Desai RH (2011) The neurobiology of semantic memory. Trends Cogn Sci 15:527-536.

Binder JR, Desai RH, Graves WW, Conant LL (2009) Where is the semantic system? A critical review and meta-analysis of 120 functional neuroimaging studies. Cereb Cortex 19:2767-2796.

Binder JR, Conant LL, Humphries CJ, Fernandino L, Simons SB, Aguilar M, Desai RH (2016) Toward a brain-based componential semantic representation. Cogn Neuropsychol 33:130-174.

Bruffaerts R, De Deyne S, Meersmans K, Liuzzi AG, Storms G, Vandenberghe $\mathrm{R}$ (2019) Redefining the resolution of semantic knowledge in the brain: advances made by the introduction of models of semantics in neuroimaging. Neurosci Biobehav Rev 103:3-13.

Bruni E, Tran N, Baroni M (2014) Multimodal distributional semantics. J Artif Intell Res 49:1-47.

Brysbaert M, New B (2009) Moving beyond Kucera and Francis: a critical evaluation of current word frequency norms and the introduction of a new and improved word frequency measure for American English. Behav Res Methods 41:977-990.

Brysbaert M, Warriner AB, Kuperman V (2014) Concreteness ratings for 40 thousand generally known English word lemmas. Behav Res Methods 46:904-911.

Bulat L, Clark S, Shutova E (2017) Speaking, seeing, understanding: correlating semantic models with conceptual representation in the brain. Proceedings of the Conference on Empirical Methods in Natural Language Processing (EMNLP 2014), pp 1532-1543. Doha, Qatar: Association for Computational Linguistics.

Chang KM, Mitchell T, Just MA (2011) Quantitative modeling of the neural representations of objects: how semantic feature norms can account for fMRI activation. Neuroimage 56:716-727.

Conneau A, Kiela D, Schwenk H, Barrault L, Bordes A (2017) Supervised learning of universal sentence representations from natural language inference data. Proceedings of the 2017 Conference on Empirical Methods in Natural Language Processing, pages 670-680, Copenhagen: Association for Computational Linguistics.

Connell L (2007) Representing object colour in language comprehension. Cognition 102:476-485.

Cox RW (1996) AFNI: software for analysis and visualization of functional magnetic resonance neuroimages. Comput Biomed Res 29:162-173.

Cree GS, McRae K (2003) Analyzing the factors underlying the structure and computation of the meaning of chipmunk, cherry, chisel, cheese, and cello (and many other such concrete nouns). J Exp Psychol Gen 132:163201.

de Heer WA, Huth AG, Griffiths TL, Gallant JL, Theunissen FE (2017) The hierarchical cortical organization of human speech processing. J Neurosci 37:6539-6557.

Devlin J, Chang MW, Lee K, Toutanova K (2019) BERT: Pre-training of Deep Bidirectional Transformers for Language Understanding. Proceedings of the 2019 Conference of the North American Chapter of the Association for Computational Linguistics: Human Language Technologies, Vol 1 (Long and Short Papers), pp 4171-4186. Minneapolis, Minnesota, June.

Dove G (2014) Thinking in words: language as an embodied medium of thought. Top Cogn Sci 6:371-389.

Dunlap WP, Cortina JM, Vaslow JB, Burke MJ (1996) Meta-analysis of experiments with matched groups or repeated measures designs. Psychol Methods 1:170.

Fedorenko E, Thompson-Schill SL (2014) Reworking the language network. Trends Cogn Sci 18:120-126.

Fernandino L, Conant LL, Binder JR, Blindauer K, Hiner B, Spangler K, Desai RH (2013) Where is the action? Action sentence processing in Parkinson's disease. Neuropsychologia 51:1510-1517.

Fernandino L, Humphries CJ, Seidenberg MS, Gross WL, Conant LL, Binder JR (2015) Predicting brain activation patterns associated with individual lexical concepts based on five sensory-motor attributes. Neuropsychologia 76:17-26.

Fernandino L, Humphries CJ, Conant LL, Seidenberg MS, Binder JR (2016) Heteromodal cortical areas encode sensory-motor features of word meaning. J Neurosci 36:9763-9769.

Fu R, Guo J, Qin B, Che W, Wang H, Liu T (2014) Learning semantic hierarchies via word embeddings. Proceedings of the 52nd Annual Meeting of 
the Association for Computational Linguistics, pp 1199-1209. Baltimore: Association for Computational Linguistics.

Fyshe A, Talukdar PP, Murphy B, Mitchell TM (2014) Interpretable semantic vectors from a joint model of brain- and text-based meaning. Proceedings of the Meeting of the Association for Computational Linguistics, pp 489-499. Baltimore: Association for Computational Linguistics.

Glasgow K, Roos M, Haufler A, Chevillet M, Wolmetz M (2016) Evaluating Semantic Models With Word-Sentence Relatedness. arXiv:1603.07253.

Glenberg AM (2010) Embodiment as a unifying perspective for psychology. Wiley Interdiscip Rev Cogn Sci 1:586-596.

Glenberg AM, Kaschak MP (2002) Grounding language in action. Psychonom Bull Rev 9:558-565.

Glenberg AM, Sato M, Cattaneo L, Riggio L, Palumbo D, Buccino G (2008) Processing abstract language modulates motor system activity. Q J Exp Psychol (Hove) 61:905-919.

Guntupalli JS, Hanke M, Halchenko YO, Connolly AC, Ramadge PJ, Haxby JV (2016) A model of representational spaces in human cortex. Cereb Cortex 26:2919-2934.

Hamilton LS, Huth AG (2018) The revolution will not be controlled: natural stimuli in speech neuroscience. Lang Cogn Neurosci 21:1-10.

Harnad S (1990) The symbol grounding problem. Phys D Nonlin Phenomena 42:335-346.

Hassabis D, Kumaran D, Summerfield C, Botvinick M (2017) Neuroscienceinspired artificial intelligence. Neuron 95:245-258.

Hasson U, Egidi G, Marelli M, Willems RM (2018) Grounding the neurobiology of language in first principles: the necessity of non-languagecentric explanations for language comprehension. Cognition 180:135157.

Haxby JV, Guntupalli JS, Connolly AC, Halchenko YO, Conroy BR, Gobbini MI, Hanke M, Ramadge PJ (2011) A common, high-dimensional model of the representational space in human ventral temporal cortex. Neuron 72:404-416.

Hoerl AE, Kennard RW (1970) Ridge regression: biased estimation for nonorthogonal problems. Technometrics 12:55-67.

Huth AG, de Heer WA, Griffiths TL, Theunissen FE, Gallant JL (2016a) Natural speech reveals the semantic maps that tile human cerebral cortex. Nature 532:453-458.

Huth AG, Lee T, Nishimoto S, Bilenko NY, Vu AT, Gallant JL (2016b) Decoding the semantic content of natural movies from human brain activity. Front Syst Neurosci 10:81.

Just MA, Cherkassky VL, Aryal S, Mitchell TM (2010) A neurosemantic theory of concrete noun representation based on the underlying brain codes. PLoS One 5:e8622.

Kaschak MP, Madden CJ, Therriault DJ, Yaxley RH, Aveyard M, Blanchard AA, Zwaan RA (2005) Perception of motion affects language processing. Cognition 94:B79-B89.

Kaschak MP, Zwaan RA, Aveyard M, Yaxley RH (2006) Perception of auditory motion affects language processing. Cogn Sci 30:733-744.

Kiela D, Clark S (2014) A systematic study of semantic vector space model parameters. Proceedings of the 2nd Workshop on Continuous Vector Space Models and their Compositionality at EACL, pp 21-30. Gothenburg, Sweden.

Kiela D, Clark S (2017) Learning Neural Audio Embeddings for Grounded Semantics in Auditory Perception. Journal of Artificial Intelligence Research 60:1003-1030.

Kousta ST, Vigliocco G, Vinson DP, Andrews M, Del Campo E (2011) The representation of abstract words: why emotion matters. J Exp Psychol Gen 140:14-34.

Kriegeskorte N, Mur M, Bandettini P (2008) Representational similarity analysis: connecting the branches of systems neuroscience. Front Syst Neurosci 2:4.

Landauer T, Dumais S (1997) A solution to Plato's problem: the latent semantic analysis theory of acquisition, induction, and representation of knowledge. Psychol Rev 104:211-240.

Louwerse MM (2018) Knowing the meaning of a word by the linguistic and perceptual company it keeps. Top Cogn Sci 10:573-589.

Louwerse MM, Jeuniaux P (2010) The linguistic and embodied nature of conceptual processing. Cognition 114:96-104.

Lund K, Burgess C (1996) Producing high-dimensional semantic spaces from lexical co-occurrence. Behav Res Methods Instrum Comput 28:203208.
Lynott D, Connell L (2013) Modality exclusivity norms for 400 nouns: the relationship between perceptual experience and surface word form. Behav Res Methods 45:516-526.

Mahon BZ (2015) What is embodied about cognition? Lang Cogn Neurosci 30:420-429.

Mahon BZ, Caramazza A (2008) A critical look at the embodied cognition hypothesis and a new proposal for grounding conceptual content. J Physiol Paris 102:59-70.

Mikolov T, Chen K, Corrado G, Dean J (2013) Efficient estimation of word representations in vector space. International Conference on Learning Representations (ICLR) Workshop, Scottsdale, AZ.

Mitchell J, Lapata M (2010) Composition in distributional models of semantics. Cogn Sci 34:1388-1429.19.

Mitchell TM, Shinkareva SV, Carlson A, Chang KM, Malave VL, Mason RA, Just MA (2008) Predicting human brain activity associated with the meaning of nouns. Science 320:1191-1195.

Oldfield RC (1971) The assessment and analysis of handedness: the Edinburgh Inventory. Neuropsychologia 9:97-113.

Paivio A (1971) Imagery and verbal processes. New York: Holt, Rinehart, and Winston.

Patterson K, Nestor PJ, Rogers TT (2007) Where do you know what you know? The representation of semantic knowledge in the human brain. Nat Rev Neurosci 8:976-987.

Pennington J, Socher R, Manning CD (2014) GloVe: global vectors for word representation. Proceedings of the 2014 Conference on Empirical Methods in Natural Language Processing, pp 1532-1543. Doha, Qatar: Association for Computational Linguistics.

Pereira F, Botvinick M, Detre G (2013) Using Wikipedia to learn semantic feature representations of concrete concepts in neuroimaging experiments. Artif Intell 194:240-252.

Pereira F, Lou B, Pritchett B, Ritter S, Gershman SJ, Kanwisher N, Botvinick M, Fedorenko E (2018) Toward a universal decoder of linguistic meaning from brain activation. Nat Commun 9:963.

Peters ME, Neumann M, Iyyer M, Gardner M, Clark C, Lee K, Zettlemoyer L (2018) Deep contextualized word representations. In Proceedings of Conference of the North American Chapter of the Association for Computational Linguistics, pp 2227-2237. Human Language Technologies, New Orleans, Louisiana.

Popov V, Ostarek M, Tenison C (2018) Practices and pitfalls in inferring neural representations. Neuroimage 174:340-351.

Pulvermüller F (2013) How neurons make meaning: brain mechanisms for embodied and abstract-symbolic semantics. Trends Cogn Sci 17:458470.

Ralph MA, Jefferies E, Patterson K, Rogers TT (2017) The neural and computational bases of semantic cognition. Nat Rev Neurosci 18:42-55.

Riordan B, Jones MN (2011) Redundancy in perceptual and linguistic experience: comparing feature based and distributional models of semantic information. Top Cogn Sci 3:303-345.

Roller S, Erk K, Boleda G (2014) Inclusive yet selective: Supervised distributional hypernymy detection. Proceedings of COLING 25th International Conference on Computational Linguistics: Technical Papers, pp 10251036. Dublin, Ireland.

Speed LJ, Vigliocco G (2014) Eye movements reveal the dynamic simulation of speed in language. Cogn Sci 38:367-382.

Stanfield RA, Zwaan RA (2001) The effect of implied orientation derived from verbal context on picture recognition. Psychol Sci 12:153-156.

Subramanian S, Trischler A, Bengio Y, Pal CJ (2018) Learning general purpose distributed sentence representations via large scale multi-task learning. Proceedings of the 2018 International Conference on Learning Representations. Vancouver, Canada.

Sudre G, Pomerleau D, Palatucci M, Wehbe L, Fyshe A, Salmelin R, Mitchell $\mathrm{T}$ (2012) Tracking neural coding of perceptual and semantic features of concrete nouns. Neuroimage 62:451-463.

Sun J, Wang S, Zhang J, Zong C (2019) Towards sentence-level brain decoding with distributed representations. Proceedings of the AAAI Conference on Artificial Intelligence, pp 7047-7054. Honolulu, Hawaii.

Talairach J, Tournoux P (1988) Co-planar stereotaxic atlas of the human brain: 3-dimensional proportional system: an approach to cerebral imaging. New York: Thieme.

Utsumi A (2018) A neurobiologically motivated analysis of distributional 
semantic models. In Proceedings of the 40th Annual Conference of the Cognitive Science Society 2018, pp 1147-1152. Madison, Wisconsin.

Vigliocco G, Meteyard L, Andrews M, Kousta S (2009) Toward a theory of semantic representation. Lang Cogn 1:219-247.

Vigliocco G, Kousta ST, Della Rosa PA, Vinson DP, Tettamanti M, Devlin JT, Cappa SF (2014) The neural representation of abstract words: the role of emotion. Cereb Cortex 24:1767-1777.

Vinson DP, Vigliocco G, Cappa S, Siri S (2003) The breakdown of semantic knowledge: insights from a statistical model of meaning representation. Brain Lang 86:347-365.

Wang J, Cherkassky VL, Just MA (2017) Predicting the brain activation pattern associated with the propositional content of a sentence: modeling neural representations of events and states. Hum Brain Mapp 38:4865-4881.

Wang X, Wu W, Ling Z, Xu Y, Fang Y, Wang X, Binder JR, Men W, Gao JH, Bi Y (2018) Organizational principles of abstract words in the human brain. Cereb Cortex 28:4305-4318.

Wehbe L, Murphy B, Talukdar P, Fyshe A, Ramdas A, Mitchell T (2014)
Simultaneously uncovering the patterns of brain regions involved in different story reading subprocesses. PLoS One 9:e112575.

Willems RM, Toni I, Hagoort P, Casasanto D (2010) Neural dissociations between action verb understanding and motor imagery. J Cogn Neurosci 22:2387-2400.

Winter B, Bergen B (2012) Language comprehenders represent object distance both visually and auditorily. Lang Cogn 4:1-16.

Yang Y, Wang J, Bailer C, Cherkassky V, Just MA (2017) Commonality of neural representations of sentences across languages: predicting brain activation during Portuguese sentence comprehension using an Englishbased model of brain function. Neuroimage 146:658-666.

Zwaan RA (2014) Embodiment and language comprehension: reframing the discussion. Trends Cogn Sci 18:229-234.

Zwaan RA, Pecher D (2012) Revisiting mental simulation in language comprehension: six replication attempts. PLoS One 7:e51382.

Zwaan RA, Stanfield RA, Yaxley RH (2002) Language comprehenders mentally represent the shapes of objects. Psychol Sci 13:168-171. 